NUB-3081-93TH

hep-ph 9311340

November 1993

(revised June 1994)

\title{
Two-Loop Renormalization Group Equations for Soft Supersymmetry-Breaking Couplings
}

\author{
Stephen P. Martin and Michael T. Vaughn \\ Department of Physics \\ Northeastern University \\ Boston MA 02115
}

\begin{abstract}
We compute the two-loop renormalization group equations for all soft supersymmetry-breaking couplings in a general softly broken $N=1$ supersymmetric model. We also specialize these results to the Minimal Supersymmetric Standard Model.
\end{abstract}




\section{Introduction}

In the Standard Model, the mass of the Higgs scalar boson is subject to quadratically divergent radiative corrections which ought to be of order $\delta m_{H}^{2} \sim \Lambda^{2}$, where $\Lambda$ is an ultraviolet cutoff scale. This presents a naturalness problem if $\Lambda^{2} \gg m_{H}^{2}$, since fine tuning is then necessary to explain why the Higgs mass is near the electroweak scale. Low-energy supersymmetry[1] evades this naturalness problem because the quadratic divergences cancel. To accomplish this, the Minimal Supersymmetric Standard Model (MSSM) introduces a "sparticle" partner for each standard model particle. If supersymmetry were exact each sparticle would be degenerate in mass with its standard model partner, which is certainly not the case experimentally, so supersymmetry must be broken. Fortunately, the cancellation of quadratic divergences still works if supersymmetry is broken softly[2] by terms of dimension 2 and 3 in the Lagrangian. The masses of the sparticles are then determined by the soft supersymmetry-breaking terms.

Because low-energy supersymmetry is a perturbative solution to the naturalness problem, one can attempt to relate observed phenomena at low energies to physics at very high energy scales. For example, it is remarkable that in the MSSM, the three gauge couplings appear to unify[3] at a scale $10^{15}-10^{16} \mathrm{GeV}$, hinting at a Grand Unified Theory (GUT) or some other organizing principle such as string theory. With the eventual discovery of the sparticles and determination of their masses, we should gain information about the soft supersymmetry-breaking parameters. Already we know that these parameters are not at all arbitrary, because otherwise large flavor-changing neutral currents would arise in the low-energy physics due to the effects of loops containing squarks, and the arbitrary complex phases of the soft supersymmetry-breaking terms would be expected to give rise to a CP-violating electric dipole moment for the neutron in violation of experimental bounds. Thus there is strong circumstantial evidence in favor of some organizing principle governing the soft supersymmetry-breaking terms.

Models obtained from supergravity[4] can provide just such an organizing principle for the soft supersymmetry-breaking terms specified at some very high input scale. In 
minimal supergravity, the spinless particles in the theory all obtain a common mass $m_{0}$ at this input scale. In this scenario, the absence of large flavor-changing neutral currents can be ascribed to the consequent near-degeneracy of the squarks. There are also scalar trilinear couplings among the squarks, sleptons and Higgs scalars as allowed by $R$-parity and gauge invariance; these are each equal at the input scale to the corresponding Yukawa coupling times a universal mass parameter $A$. There is also a supersymmetry-breaking scalar $(\mathrm{mass})^{2}$ in the Higgs sector. Finally, there are three gaugino masses which also break supersymmetry. Large CP violation can be avoided with a common complex phase for all of the soft supersymmetry-breaking parameters. If one makes further assumptions about the high energy physics, additional constraints on the parameters of the theory are obtained. For example, if there is a GUT, then the gauge couplings and gaugino masses are unified, and there may be relations among the Yukawa couplings and among the scalar trilinear interactions at the unification scale. However, since the supersymmetry-breaking mechanism remains mysterious, a precise formulation of the organizing principle behind the soft terms in the MSSM remains unclear.

In any case, with the parameters of the model specified at the input scale by some candidate organizing principle, one can run the couplings and masses down to low energies using the renormalization group equations and make predictions about the sparticle masses and other low-energy phenomena. Many authors (for example, [5-17]) have provided numerical and analytical results for the sparticle spectrum under a variety of assumptions and constraints on the input parameters. In studies of this type, greater precision as well as an estimate of errors incurred in the running can be obtained by employing the twoloop renormalization group equations. The two-loop $\beta$-functions for the supersymmetric couplings (gauge couplings[18] and superpotential parameters[19]) have been known for some time. Recently, the two-loop $\beta$-functions for gaugino mass parameters have also been found[20,21]. In this paper, we will complete the list of two-loop $\beta$-functions for a general softly-broken supersymmetric model, by computing the results for scalar interactions which break supersymmetry softly. We will also give the results of specializing these calculations to the MSSM. 
We consider a general $N=1$ supersymmetric Yang-Mills model. The chiral superfields $\Phi_{i}$ contain a complex scalar $\phi_{i}$ and a two-component fermion $\psi_{i}$ which transform as a (possibly reducible) representation $R$ of the gauge group $G$. The superpotential is

$$
W=\frac{1}{6} Y^{i j k} \Phi_{i} \Phi_{j} \Phi_{k}+\frac{1}{2} \mu^{i j} \Phi_{i} \Phi_{j}+L^{i} \Phi_{i}
$$

In addition, the Lagrangian contains soft supersymmetry-breaking terms of the form

$$
\mathcal{L}_{S B}=-\frac{1}{6} h^{i j k} \phi_{i} \phi_{j} \phi_{k}-\frac{1}{2} b^{i j} \phi_{i} \phi_{j}-\frac{1}{2}\left(m^{2}\right)_{i}^{j} \phi^{* i} \phi_{j}-\frac{1}{2} M \lambda \lambda+\text { h.c. }
$$

where $M$ is the mass of the gaugino $\lambda$. The renormalization group equations for the gauge coupling and the superpotential parameters $Y^{i j k}, \mu^{i j}$ and $L^{i}$ and the gaugino mass $M$ are known. In this paper we give the corresponding results for the soft-breaking parameters $h^{i j k}, b^{i j}$, and $\left(m^{2}\right)_{i}^{j}$.

For simplicity we first give our results for the special case of a simple [or $U(1)$ ] gauge group. We then explain the modifications required if the gauge group is a direct product in Section III, and discuss the specialization of these results to the MSSM in Section IV. We let $\mathbf{t}^{A} \equiv(\mathbf{t})_{i}^{A j}$ denote the representation matrices for the gauge group $G$. Then

$$
\begin{aligned}
\left(\mathbf{t}^{A} \mathbf{t}^{A}\right)_{i}^{j} & \equiv C(R) \delta_{i}^{j} \\
\operatorname{Tr}_{R}\left(\mathbf{t}^{A} \mathbf{t}^{B}\right) & \equiv S(R) \delta^{A B}
\end{aligned}
$$

define the quadratic Casimir invariant $C(R)$ and the Dynkin index $S(R)$ for the representation $R$. For the adjoint representation [of dimension denoted by $d(G)$ ] $C(G) \delta^{A B}=$ $f^{A C D} f^{B C D}$ with $f^{A B C}$ the structure constants of the group.

In principle, the two-loop $\beta$-functions for a general renormalizable theory have already been given in $[22,23,24]$. However, there are two practical issues which must be addressed in order to apply those results to the case at hand.

The first issue is that only dimensionless couplings appeared in [22-24]. The two-loop $\beta$-function for a gauge coupling was given in [22], for a Yukawa coupling in [23], and for a scalar quartic coupling in [24]. A general renormalizable theory may also contain 
fermion mass terms, scalar (mass) ${ }^{2}$ terms, and trilinear scalar couplings. Fortunately, the $\beta$-functions for each of these dimensionful couplings can be inferred from the results given in [23] and [24] by taking some of the scalar fields to be non-propagating "dummy" fields with no gauge interactions. For example, a fermion mass term has the form

$$
\mathcal{L}_{\mathcal{M}}=-\mathcal{M}^{i j} \psi_{i} \psi_{j}=-\varphi_{\text {dummy }} Y_{\text {dummy }}^{i j} \psi_{i} \psi_{j}
$$

Now if $\varphi_{\text {dummy }}$ is taken to have no other interactions, then the $\beta$-function for the fermion mass $\mathcal{M}^{i j}$ has the same form as that of the Yukawa coupling $Y_{\text {dummy }}^{i j}$. Thus the $\beta$-function for any fermion mass can be inferred directly from the results of [23]. Similarly, scalar (mass) $)^{2}$ terms can always be thought of as scalar quartic interactions involving two dummy scalars and two normal scalars, while scalar trilinear couplings can be thought of as scalar quartic interactions with one dummy scalar and three normal scalars, so the two-loop $\beta$ functions for those cases can be inferred from the results of [24] using a small amount of careful combinatorics associated with the symmetry factors.

The second issue to be addressed is that the results of [22-24] were obtained using dimensional regularization [25] (DREG). Now, DREG violates supersymmetry explicitly because it introduces a mismatch between the numbers of gauge boson and gaugino degrees of freedom. Therefore the use of DREG is inappropriate for (even softly broken) supersymmetric models. Instead, one should use the modified scheme known as dimensional reduction [26] (DRED) which does not violate supersymmetry.

In DREG, supersymmetry is violated in the finite parts of one-loop graphs, and in the divergent parts of two-loop graphs. This means that for a given set of physical quantities (e.g. pole masses and S-matrix elements) the running couplings computed in DREG with modified minimal subtraction[27] ( $\overline{\mathrm{MS}})$ will differ from those computed in DRED with modified minimal subtraction $(\overline{\mathrm{DR}})$ by finite one-loop corrections, and the $\beta$-functions will be different for the two schemes starting at the two-loop level. We therefore present our $\beta$-functions as they appear in the $\overline{\mathrm{DR}}$ scheme. This means that the results of [22-24] must be translated from $\overline{\mathrm{MS}}$ to $\overline{\mathrm{DR}}$. In [20], we provided a "dictionary" for translating couplings between the two schemes including all finite one-loop radiative corrections, which is the 
order necessary for the present application to two-loop $\beta$-functions. (The relationship between DRED and DREG for non-supersymmetric theories has recently been illuminated in [28].) We must also take into account the fact that in DRED, the so-called $\epsilon$-scalars[26] obtain one-loop mass counterterms due to the supersymmetry-breaking scalar and gaugino masses.

In summary, our method of computation is to use the results of [22-24] for a general renormalizable theory, using dummy scalars where necessary for dimensionful couplings, and translating the results from $\overline{\mathrm{MS}}$ to $\overline{\mathrm{DR}}$. This process is straightforward but rather tedious, and we decline to exhibit the details here. It may also be possible in principle to extract these results from the dimensional reduction calculations of [29].

\section{Two-Loop Running in a General Softly Broken Supersymmetric Model}

For completeness, we begin by reviewing the known results for gauge couplings, gaugino masses, and superpotential parameters (including Yukawa couplings). This will also serve as a useful point of reference for the notation established in the Introduction. We will

then provide the two-loop beta functions for $h^{i j k}, b^{i j}$, and $\left(m^{2}\right)_{i}^{j}$, which constitute the new results of this paper. We do not assume anything about the relative complex phases of any of the parameters.

The gauge coupling at two loops is actually scheme-independent. Therefore, it may be obtained simply by specializing the results of [22] to a general supersymmetric model. Doing so, we obtain the known[18] result:

$$
\begin{aligned}
& \frac{d}{d t} g=\frac{1}{16 \pi^{2}} \beta_{g}^{(1)}+\frac{1}{\left(16 \pi^{2}\right)^{2}} \beta_{g}^{(2)} \\
\beta_{g}^{(1)}= & g^{3}[S(R)-3 C(G)] \\
\beta_{g}^{(2)}= & g^{5}\left\{-6[C(G)]^{2}+2 C(G) S(R)+4 S(R) C(R)\right\}-g^{3} Y^{i j k} Y_{i j k} C(k) / d(G) .
\end{aligned}
$$

Here $Y_{i j k}=\left(Y^{i j k}\right)^{*}$ and $S(R)$ is the Dynkin index summed over all chiral multiplets and $S(R) C(R)$ is the sum of the Dynkin indices weighted by the quadratic Casimir invariant. 
The two-loop $\beta$-function for the gaugino mass parameter may be computed (as we did in [20]) by first using the results of [23] for a general theory in $\overline{\mathrm{MS}}$ and then translating the results to the $\overline{\mathrm{DR}}$ scheme appropriate for supersymmetry. The result is:

$$
\begin{aligned}
& \frac{d}{d t} M=\frac{1}{16 \pi^{2}} \beta_{M}^{(1)}+\frac{1}{\left(16 \pi^{2}\right)^{2}} \beta_{M}^{(2)} \\
& \beta_{M}^{(1)}= g^{2}[2 S(R)-6 C(G)] M \\
& \beta_{M}^{(2)}=g^{4}\left\{-24[C(G)]^{2}+8 C(G) S(R)+16 S(R) C(R)\right\} M \\
& \\
& \quad+2 g^{2}\left[h^{i j k}-M Y^{i j k}\right] Y_{i j k} C(k) / d(G) .
\end{aligned}
$$

This result was also obtained by Y. Yamada[21] using a different method. Note that we have adopted a slightly different notation here than we did in [20], since the heights of indices on the last term in (2.6) have been reversed. This is the same as exchanging the role of the gaugino mass $M$ and its conjugate $M^{\dagger}$. We do this to ensure consistency with results below while maintaining the fewest possible number of $M^{\dagger}$ s.

The two-loop $\beta$-functions for the superpotential parameters can be obtained either by superfield techniques[19], or by applying the general results of [23] and [24]. In applying the latter method, one must be careful to convert the $\overline{\mathrm{MS}}$ results into $\overline{\mathrm{DR}}$, as we have already mentioned. We obtain the same results using both methods:

$$
\begin{aligned}
\frac{d}{d t} Y^{i j k} & =Y^{i j p}\left[\frac{1}{16 \pi^{2}} \gamma_{p}^{(1) k}+\frac{1}{\left(16 \pi^{2}\right)^{2}} \gamma_{p}^{(2) k}\right]+(k \leftrightarrow i)+(k \leftrightarrow j) \\
\frac{d}{d t} \mu^{i j} & =\mu^{i p}\left[\frac{1}{16 \pi^{2}} \gamma_{p}^{(1) j}+\frac{1}{\left(16 \pi^{2}\right)^{2}} \gamma_{p}^{(2) j}\right]+(j \leftrightarrow i) \\
\frac{d}{d t} L^{i} & =L^{p}\left[\frac{1}{16 \pi^{2}} \gamma_{p}^{(1) i}+\frac{1}{\left(16 \pi^{2}\right)^{2}} \gamma_{p}^{(2) i}\right]
\end{aligned}
$$

where

$$
\begin{aligned}
& \gamma_{i}^{(1) j}= \frac{1}{2} Y_{i p q} Y^{j p q}-2 \delta_{i}^{j} g^{2} C(i) \\
& \gamma_{i}^{(2) j}=-\frac{1}{2} Y_{i m n} Y^{n p q} Y_{p q r} Y^{m r j}+g^{2} Y_{i p q} Y^{j p q}[2 C(p)-C(i)] \\
& \quad+2 \delta_{i}^{j} g^{4}\left[C(i) S(R)+2 C(i)^{2}-3 C(G) C(i)\right] .
\end{aligned}
$$

In these equations, $C(r)$ always refers to the quadratic Casimir invariant of the representation carried by the indicated chiral superfield, while $S(R)$ refers to the total Dynkin index 
summed over all of the chiral superfields. The objects $\gamma_{i}^{(1) j}$ and $\gamma_{i}^{(2) j}$ arise completely from wave-function renormalization in the superfield approach, in accordance with the "non-renormalization" theorems of supersymmetry[30].

Next we consider the two-loop $\beta$-function for the soft supersymmetry-breaking scalar trilinear coupling $h^{i j k}$. This is obtained by specializing eqs. (4.3) and (4.7) of [24] to the supersymmetric case, with one of the external scalar fields being a dummy field, and then translating the result from $\overline{\mathrm{MS}}$ to $\overline{\mathrm{DR}}$ using [20]. The result is:

$$
\begin{aligned}
\frac{d}{d t} h^{i j k}= & \frac{1}{16 \pi^{2}}\left[\beta_{h}^{(1)}\right]^{i j k}+\frac{1}{\left(16 \pi^{2}\right)^{2}}\left[\beta_{h}^{(2)}\right]^{i j k} \\
{\left[\beta_{h}^{(1)}\right]^{i j k}=} & \frac{1}{2} h^{i j l} Y_{l m n} Y^{m n k}+Y^{i j l} Y_{l m n} h^{m n k}-2\left(h^{i j k}-2 M Y^{i j k}\right) g^{2} C(k) \\
& +(k \leftrightarrow i)+(k \leftrightarrow j) \\
{\left[\beta_{h}^{(2)}\right]^{i j k}=} & -\frac{1}{2} h^{i j l} Y_{l m n} Y^{n p q} Y_{p q r} Y^{m r k} \\
& -Y^{i j l} Y_{l m n} Y^{n p q} Y_{p q r} h^{m r k}-Y^{i j l} Y_{l m n} h^{n p q} Y_{p q r} Y^{m r k} \\
& +\left(h^{i j l} Y_{l p q} Y^{p q k}+2 Y^{i j l} Y_{l p q} h^{p q k}-2 M Y^{i j l} Y_{l p q} Y^{p q k}\right) g^{2}[2 C(p)-C(k)] \\
& +\left(2 h^{i j k}-8 M Y^{i j k}\right) g^{4}\left[C(k) S(R)+2 C(k)^{2}-3 C(G) C(k)\right] \\
& +(k \leftrightarrow i)+(k \leftrightarrow j) .
\end{aligned}
$$

Next we consider the two-loop $\beta$-function for the scalar (mass) ${ }^{2} b^{i j}$. This is again obtained by specializing eqs. (4.3) and (4.7) of [24] to the supersymmetric case, but with two of the external scalar fields as dummy fields, and then translating the result from $\overline{\mathrm{MS}}$ to $\overline{\mathrm{DR}}$ using the results of [20]. Doing so, we find:

$$
\begin{aligned}
\frac{d}{d t} b^{i j}= & \frac{1}{16 \pi^{2}}\left[\beta_{b}^{(1)}\right]^{i j}+\frac{1}{\left(16 \pi^{2}\right)^{2}}\left[\beta_{b}^{(2)}\right]^{i j} \\
{\left[\beta_{b}^{(1)}\right]^{i j}=} & \frac{1}{2} b^{i l} Y_{l m n} Y^{m n j}+\frac{1}{2} Y^{i j l} Y_{l m n} b^{m n}+\mu^{i l} Y_{l m n} h^{m n j}-2\left(b^{i j}-2 M \mu^{i j}\right) g^{2} C(i) \\
& +(i \leftrightarrow j) \\
{\left[\beta_{b}^{(2)}\right]^{i j}=} & -\frac{1}{2} b^{i l} Y_{l m n} Y^{p q n} Y_{p q r} Y^{m r j}-\frac{1}{2} Y^{i j l} Y_{l m n} b^{m r} Y_{p q r} Y^{p q n}
\end{aligned}
$$




$$
\begin{aligned}
& -\frac{1}{2} Y^{i j l} Y_{l m n} \mu^{m r} Y_{p q r} h^{p q n}-\mu^{i l} Y_{l m n} h^{n p q} Y_{p q r} Y^{m r j} \\
& -\mu^{i l} Y_{l m n} Y^{n p q} Y_{p q r} h^{m r j}+2 Y^{i j l} Y_{l p q}\left(b^{p q}-\mu^{p q} M\right) g^{2} C(p) \\
& +\left(b^{i l} Y_{l p q} Y^{p q j}+2 \mu^{i l} Y_{l p q} h^{p q j}-2 \mu^{i l} Y_{l p q} Y^{p q j} M\right) g^{2}[2 C(p)-C(i)] \\
& +\left(2 b^{i j}-8 \mu^{i j} M\right) g^{4}\left[C(i) S(R)+2 C(i)^{2}-3 C(G) C(i)\right] \\
& +(i \leftrightarrow j) .
\end{aligned}
$$

Finally, we consider the two-loop $\beta$-function for the scalar $(\operatorname{mass})^{2}\left(m^{2}\right)_{i}^{j}$. This is once again obtained by specializing eqs. (4.3) and (4.7) of [24] to the supersymmetric case, with two of the external scalar fields as dummy fields, and translating from $\overline{\mathrm{MS}}$ to $\overline{\mathrm{DR}}$ using [20]. One must also be careful here to take into account the mass counterterms for the $\epsilon$-scalars which arise in DRED. The result is:

$$
\begin{aligned}
\frac{d}{d t}\left(m^{2}\right)_{i}^{j}= & \frac{1}{16 \pi^{2}}\left[\beta_{m^{2}}^{(1)}\right]_{i}^{j}+\frac{1}{\left(16 \pi^{2}\right)^{2}}\left[\beta_{m^{2}}^{(2)}\right]_{i}^{j} \\
{\left[\beta_{m^{2}}^{(1)}\right]_{i}^{j}=} & \frac{1}{2} Y_{i p q} Y^{p q n}\left(m^{2}\right)_{n}^{j}+\frac{1}{2} Y^{j p q} Y_{p q n}\left(m^{2}\right)_{i}^{n}+2 Y_{i p q} Y^{j p r}\left(m^{2}\right)_{r}^{q} \\
& +h_{i p q} h^{j p q}-8 \delta_{i}^{j} M M^{\dagger} g^{2} C(i)+2 g^{2} \mathbf{t}_{i}^{A j} \operatorname{Tr}\left[\mathbf{t}^{A} m^{2}\right] \\
{\left[\beta_{m^{2}}^{(2)}\right]_{i}^{j}=} & -\frac{1}{2}\left(m^{2}\right)_{i}^{l} Y_{l m n} Y^{m r j} Y_{p q r} Y^{p q n}-\frac{1}{2}\left(m^{2}\right)_{l}^{j} Y^{l m n} Y_{m r i} Y^{p q r} Y_{p q n} \\
& -Y_{i l m} Y^{j n m}\left(m^{2}\right)_{r}^{l} Y_{n p q} Y^{r p q}-Y_{i l m} Y^{j n m}\left(m^{2}\right)_{n}^{r} Y_{r p q} Y^{l p q} \\
& -Y_{i l m} Y^{j n r}\left(m^{2}\right)_{n}^{l} Y_{p q r} Y^{p q m}-2 Y_{i l m} Y^{j l n} Y_{n p q} Y^{m p r}\left(m^{2}\right)_{r}^{q} \\
& -Y_{i l m} Y^{j l n} h_{n p q} h^{m p q}-h_{i l m} h^{j l n} Y_{n p q} Y^{m p q} \\
& -h_{i l m} Y^{j l n} Y_{n p q} h^{m p q}-Y_{i l m} h^{j l n} h_{n p q} Y^{m p q} \\
& +\left[\left(m^{2}\right)_{i}^{l} Y_{l p q} Y^{j p q}+Y_{i p q} Y^{l p q}\left(m^{2}\right)_{l}^{j}+4 Y_{i p q} Y^{j p l}\left(m^{2}\right)_{l}^{q}+2 h_{i p q} h^{j p q}\right. \\
& \left.-2 h_{i p q} Y^{j p q} M-2 Y_{i p q} h^{j p q} M^{\dagger}+4 Y_{i p q} Y^{j p q} M M^{\dagger}\right] g^{2}[C(p)+C(q)-C(i)] \\
& -2 g^{2} \mathbf{t}_{i}^{A j}\left(\mathbf{t}^{A} m^{2}\right)_{r}^{l} Y_{l p q} Y^{r p q}+8 g^{4} \mathbf{t}_{i}^{A j} \operatorname{Tr}\left[\mathbf{t}^{A} C(r) m^{2}\right] \\
& +\delta_{i}^{j} g^{4} M M^{\dagger}\left[24 C(i) S(R)+48 C(i)^{2}-72 C(G) C(i)\right] \\
& +8 \delta_{i}^{j} g^{4} C(i)\left(\operatorname{Tr}\left[S(r) m^{2}\right]-C(G) M M^{\dagger}\right) .
\end{aligned}
$$

Here $h_{i j k}=\left(h^{i j k}\right)^{*}$. The traces are over all of the chiral superfields, and the $C(r)$ are the 
quadratic Casimir invariants for the irreducible representations of chiral superfields in the

traces. Note that the terms which explicitly involve $\mathbf{t}_{i}^{A j}$ are zero for non-abelian groups.

This completes the list of two-loop $\beta$-functions for a general softly-broken supersymmetric theory. We conclude this section by noting a non-trivial consistency check on these results. It has been shown[31] that an $N=2$ supersymmetric Yang-Mills theory is finite to all orders in perturbation theory provided that certain constraints are imposed on the representations of superfields. This finiteness continues to hold even with soft breaking, provided that the soft terms obey certain additional constraints[32]. It is an amusing exercise to check that when the above formulas for a general $N=1$ supersymmetric model are specialized to the $N=2$ case with the appropriate contraints on the representations and soft couplings, the two-loop $\beta$-functions do indeed vanish.

\section{Two-Loop Beta Functions for Direct Product Groups}

As promised, we now point out the modifications which must be made to the preceding formulas if the gauge group is a product of simple [or $U(1)$ ] subgroups $G_{a}$.

One obtains the $\beta$-function for each gauge coupling $g_{a}$ by applying the following rules to $(2.2)-(2.3)$ :

$$
\begin{aligned}
g^{3} C(G) & \rightarrow g_{a}^{3} C\left(G_{a}\right) \\
g^{3} S(R) & \rightarrow g_{a}^{3} S_{a}(R) \\
g^{5} C(G)^{2} & \rightarrow g_{a}^{5} C\left(G_{a}\right)^{2} \\
g^{5} C(G) S(R) & \rightarrow g_{a}^{5} C\left(G_{a}\right) S_{a}(R) \\
g^{5} S(R) C(R) & \rightarrow \sum_{b} g_{a}^{3} g_{b}^{2} S_{a}(R) C_{b}(R) \\
g^{3} C(k) / d(G) & \rightarrow g_{a}^{3} C_{a}(k) / d\left(G_{a}\right) .
\end{aligned}
$$

The $\sum_{b}$ in (3.5) is a sum over subgroups. Similarly, one obtains the $\beta$-function for each gaugino mass parameter $M_{a}$ by applying to (2.5)-(2.6) the same rules as above, but with 
one less power of $g \rightarrow g_{a}$, and with $M \rightarrow M_{a}$ wherever it appears. The exception is that

$$
16 g^{4} S(R) C(R) M \rightarrow 8 \sum_{b} g_{a}^{2} g_{b}^{2} S_{a}(R) C_{b}(R)\left(M_{a}+M_{b}\right)
$$

in (2.6). Note that in these cases, all terms which do not contain a quadratic Casimir invariant of a non-adjoint representation are simply diagonal in each subgroup.

For the $\beta$-functions of the superpotential parameters and soft supersymmetry-breaking scalar interactions (2.7)-(2.20), one always obtains a sum over subgroups. In each of these cases, the following set of rules apply:

$$
\begin{aligned}
g^{2} C(r) & \rightarrow \sum_{a} g_{a}^{2} C_{a}(r) \\
g^{4} C(r) S(R) & \rightarrow \sum_{a} g_{a}^{4} C_{a}(r) S_{a}(R) \\
g^{4} C(r) C(G) & \rightarrow \sum_{a} g_{a}^{4} C_{a}(r) C\left(G_{a}\right) \\
g^{4} C(r)^{2} & \rightarrow \sum_{a} \sum_{b} g_{a}^{2} g_{b}^{2} C_{a}(r) C_{b}(r) .
\end{aligned}
$$

Terms which also involve gaugino masses are modified exactly as above, with

$$
M, M^{\dagger} \rightarrow M_{a}, M_{a}^{\dagger}
$$

except for the term

$$
48 g^{4} M M^{\dagger} C(i)^{2} \rightarrow \sum_{a} \sum_{b} g_{a}^{2} g_{b}^{2} C_{a}(i) C_{b}(i)\left[32 M_{a} M_{a}^{\dagger}+8 M_{a} M_{b}^{\dagger}+8 M_{b} M_{a}^{\dagger}\right]
$$

in eq. (2.20). We also have in (2.19)-(2.20):

$$
\begin{aligned}
g^{2} \mathbf{t}_{i}^{A j} \operatorname{Tr}\left[\mathbf{t}^{A} m^{2}\right] & \rightarrow \sum_{a} g_{a}^{2}\left(\mathbf{t}_{a}^{A}\right)_{i}^{j} \operatorname{Tr}\left[\mathbf{t}_{a}^{A} m^{2}\right] \\
g^{2} \mathbf{t}_{i}^{A j}\left(\mathbf{t}^{A} m^{2}\right)_{r}^{l} Y_{l p q} Y^{r p q} & \rightarrow \sum_{a} g_{a}^{2}\left(\mathbf{t}_{a}^{A}\right)_{i}^{j}\left(\mathbf{t}_{a}^{A} m^{2}\right)_{r}^{l} Y_{l p q} Y^{r p q} \\
g^{4} \mathbf{t}_{i}^{A j} \operatorname{Tr}\left[\mathbf{t}^{A} C(r) m^{2}\right] & \rightarrow \sum_{a} \sum_{b} g_{a}^{2} g_{b}^{2}\left(\mathbf{t}_{a}^{A}\right)_{i}^{j} \operatorname{Tr}\left[\mathbf{t}_{a}^{A} C_{b}(r) m^{2}\right] \\
g^{4} C(i) \operatorname{Tr}\left[S(r) m^{2}\right] & \rightarrow \sum_{a} g_{a}^{4} C_{a}(i) \operatorname{Tr}\left[S_{a}(r) m^{2}\right] .
\end{aligned}
$$


Finally, we must note that there is an exceptional case when the gauge group contains (unlike the MSSM) a direct product of more than one $U(1)$. One should then choose the basis for the $U(1)$ subgroups so that the matrix $\operatorname{Tr}\left[q_{a} q_{b}\right]$ is diagonal, where the trace is over all chiral superfields and $q_{a}$ denotes the $U(1)_{a}$ charge. This is always possible, since $\operatorname{Tr}\left[q_{a} q_{b}\right]$ is always a real symmetric matrix. Then the only non-trivial rule is that the term $g^{4} C(i) \operatorname{Tr}\left[S(r) m^{2}\right]$ in (2.20) becomes a sum over non- $U(1)$ subgroups as before, plus a contribution

$$
g^{4} C(i) \operatorname{Tr}\left[S(r) m^{2}\right] \rightarrow \sum_{a} \sum_{b} g_{a}^{2} g_{b}^{2}\left(q_{a}\right)_{i}\left(q_{b}\right)_{i} \operatorname{Tr}\left[q_{a} q_{b} m^{2}\right]
$$

where $\sum_{a}$ and $\sum_{b}$ are sums over $U(1)$ subgroups, and $\left(q_{a}\right)_{i}$ denotes the $U(1)_{a}$ charge of the chiral superfield carrying the index $i$.

IV. Two-Loop Running in the Minimal Supersymmetric Standard Model

In the MSSM, the gauge group is $S U(3)_{c} \times S U(2)_{L} \times U(1)_{Y}$, with chiral superfields $Q$ and $L$ for the $S U(2)_{L}$-doublet quarks and leptons, and $u, d, e$ for the $S U(2)_{L}$-singlet quarks and leptons, and two Higgs doublet chiral superfields $H_{u}$ and $H_{d}$. The superpotential is

$$
W=u \mathbf{Y}_{u} Q H_{u}+d \mathbf{Y}_{d} Q H_{d}+e \mathbf{Y}_{e} L H_{d}+\mu H_{u} H_{d}
$$

where $\mathbf{Y}_{u}, \mathbf{Y}_{d}, \mathbf{Y}_{e}$ are each $3 \times 3$ Yukawa matrices. The soft supersymmetry-breaking Lagrangian contains scalar couplings

$$
-\mathcal{L}=\hat{u} \mathbf{h}_{u} \hat{Q} \hat{H}_{u}+\hat{d} \mathbf{h}_{d} \hat{Q} \hat{H}_{d}+\hat{e} \mathbf{h}_{e} \hat{L} \hat{H}_{d}+B \hat{H}_{u} \hat{H}_{d}+\text { h.c. }
$$

where $\mathbf{h}_{u}, \mathbf{h}_{d}, \mathbf{h}_{e}$ are again $3 \times 3$ matrices in family space, and a hat is used to denote the scalar component of each chiral superfield. There are also scalar masses of the $\left(m^{2}\right)_{i}^{j}$ type:

$$
-\mathcal{L}=m_{H_{u}}^{2} \hat{H}_{u}^{\dagger} \hat{H}_{u}+m_{H_{d}}^{2} \hat{H}_{d}^{\dagger} \hat{H}_{d}+\hat{Q}^{\dagger} \mathbf{m}_{Q}^{2} \hat{Q}+\hat{L}^{\dagger} \mathbf{m}_{L}^{2} \hat{L}+\hat{u} \mathbf{m}_{u}^{2} \hat{u}^{\dagger}+\hat{d} \mathbf{m}_{d}^{2} \hat{d}^{\dagger}+\hat{e} \mathbf{m}_{e}^{2} \hat{e}^{\dagger}
$$

Here again $\mathbf{m}_{Q}^{2}, \mathbf{m}_{L}^{2}, \mathbf{m}_{u}^{2}, \mathbf{m}_{d}^{2}$, and $\mathbf{m}_{e}^{2}$ are $3 \times 3$ matrices in family space. Finally, the gauginos for the subgroups $S U(3)_{c}, S U(2)_{L}$ and $U(1)_{Y}$ have masses $M_{3}, M_{2}$, and $M_{1}$, 
respectively. Again, we do not assume anything about the complex phases of any of the parameters. For most applications, it will be sufficient to retain only the Yukawa couplings of the heaviest family, but we prefer to retain complete generality.

For the sake of completeness and to provide a useful point of reference, we begin by reviewing the known results for two-loop $\beta$-functions within the MSSM. For the three gauge couplings, we have from (2.1)-(2.3):

$$
\frac{d}{d t} g_{a}=\frac{g_{a}^{3}}{16 \pi^{2}} B_{a}^{(1)}+\frac{g_{a}^{3}}{\left(16 \pi^{2}\right)^{2}}\left[\sum_{b=1}^{3} B_{a b}^{(2)} g_{b}^{2}-\sum_{x=u, d, e} C_{a}^{x} \operatorname{Tr}\left(Y_{x}^{\dagger} Y_{x}\right)\right] .
$$

Here $B_{a}^{(1)}=(33 / 5,1,-3)$ for $U(1)_{Y}$ (in a GUT normalization), $S U(2)_{L}$, and $S U(3)_{c}$ respectively, and

$$
B_{a b}^{(2)}=\left(\begin{array}{ccc}
199 / 25 & 27 / 5 & 88 / 5 \\
9 / 5 & 25 & 24 \\
11 / 5 & 9 & 14
\end{array}\right) \quad \text { and } \quad C_{a}^{u, d, e}=\left(\begin{array}{ccc}
26 / 5 & 14 / 5 & 18 / 5 \\
6 & 6 & 2 \\
4 & 4 & 0
\end{array}\right) .
$$

The two-loop renormalization group equations for the three gaugino mass parameters $[20,21]$ can then be written easily in terms of the same coefficients:

$$
\begin{aligned}
\frac{d}{d t} M_{a}=\frac{2 g_{a}^{2}}{16 \pi^{2}} B_{a}^{(1)} M_{a} & +\frac{2 g_{a}^{2}}{\left(16 \pi^{2}\right)^{2}}\left[\sum_{b=1}^{3} B_{a b}^{(2)} g_{b}^{2}\left(M_{a}+M_{b}\right)\right. \\
& \left.+\sum_{x=u, d, e} C_{a}^{x}\left(\operatorname{Tr}\left[Y_{x}^{\dagger} h_{x}\right]-M_{a} \operatorname{Tr}\left[Y_{x}^{\dagger} Y_{x}\right]\right)\right] .
\end{aligned}
$$

From (2.7)-(2.11), the two-loop beta functions for the superpotential parameters are:

$$
\begin{aligned}
\frac{d}{d t} \mu & =\frac{1}{16 \pi^{2}} \beta_{\mu}^{(1)}+\frac{1}{\left(16 \pi^{2}\right)^{2}} \beta_{\mu}^{(2)} \\
\frac{d}{d t} \mathbf{Y}_{u, d, e} & =\frac{1}{16 \pi^{2}} \beta_{\mathbf{Y}_{u, d, e}}^{(1)}+\frac{1}{\left(16 \pi^{2}\right)^{2}} \beta_{\mathbf{Y}_{u, d, e}}^{(2)}
\end{aligned}
$$

with:

$$
\begin{aligned}
\beta_{\mu}^{(1)}=\mu\{ & \left.\operatorname{Tr}\left(3 \mathbf{Y}_{u} \mathbf{Y}_{u}^{\dagger}+3 \mathbf{Y}_{d} \mathbf{Y}_{d}^{\dagger}+\mathbf{Y}_{e} \mathbf{Y}_{e}^{\dagger}\right)-3 g_{2}^{2}-\frac{3}{5} g_{1}^{2}\right\} \\
\beta_{\mu}^{(2)}=\mu\{ & -3 \operatorname{Tr}\left(3 \mathbf{Y}_{u} \mathbf{Y}_{u}^{\dagger} \mathbf{Y}_{u} \mathbf{Y}_{u}^{\dagger}+3 \mathbf{Y}_{d} \mathbf{Y}_{d}^{\dagger} \mathbf{Y}_{d} \mathbf{Y}_{d}^{\dagger}+2 \mathbf{Y}_{u} \mathbf{Y}_{d}^{\dagger} \mathbf{Y}_{d} \mathbf{Y}_{u}^{\dagger}+\mathbf{Y}_{e} \mathbf{Y}_{e}^{\dagger} \mathbf{Y}_{e} \mathbf{Y}_{e}^{\dagger}\right) \\
& +\left[16 g_{3}^{2}+\frac{4}{5} g_{1}^{2}\right] \operatorname{Tr}\left(\mathbf{Y}_{u} \mathbf{Y}_{u}^{\dagger}\right)+\left[16 g_{3}^{2}-\frac{2}{5} g_{1}^{2}\right] \operatorname{Tr}\left(\mathbf{Y}_{d} \mathbf{Y}_{d}^{\dagger}\right)+\frac{6}{5} g_{1}^{2} \operatorname{Tr}\left(\mathbf{Y}_{e} \mathbf{Y}_{e}^{\dagger}\right) \\
& \left.+\frac{15}{2} g_{2}^{4}+\frac{9}{5} g_{1}^{2} g_{2}^{2}+\frac{207}{50} g_{1}^{4}\right\}
\end{aligned}
$$




$$
\begin{aligned}
& \beta_{\mathbf{Y}_{u}}^{(1)}=\mathbf{Y}_{u}\left\{3 \operatorname{Tr}\left(\mathbf{Y}_{u} \mathbf{Y}_{u}^{\dagger}\right)+3 \mathbf{Y}_{u}^{\dagger} \mathbf{Y}_{u}+\mathbf{Y}_{d}^{\dagger} \mathbf{Y}_{d}-\frac{16}{3} g_{3}^{2}-3 g_{2}^{2}-\frac{13}{15} g_{1}^{2}\right\} \\
& \beta_{\mathbf{Y}_{u}}^{(2)}=\mathbf{Y}_{u}\left\{-3 \operatorname{Tr}\left(3 \mathbf{Y}_{u} \mathbf{Y}_{u}^{\dagger} \mathbf{Y}_{u} \mathbf{Y}_{u}^{\dagger}+\mathbf{Y}_{u} \mathbf{Y}_{d}^{\dagger} \mathbf{Y}_{d} \mathbf{Y}_{u}^{\dagger}\right)-\mathbf{Y}_{d}^{\dagger} \mathbf{Y}_{d} \operatorname{Tr}\left(3 \mathbf{Y}_{d} \mathbf{Y}_{d}^{\dagger}+\mathbf{Y}_{e} \mathbf{Y}_{e}^{\dagger}\right)\right. \\
& -9 \mathbf{Y}_{u}^{\dagger} \mathbf{Y}_{u} \operatorname{Tr}\left(\mathbf{Y}_{u} \mathbf{Y}_{u}^{\dagger}\right)-4 \mathbf{Y}_{u}^{\dagger} \mathbf{Y}_{u} \mathbf{Y}_{u}^{\dagger} \mathbf{Y}_{u}-2 \mathbf{Y}_{d}^{\dagger} \mathbf{Y}_{d} \mathbf{Y}_{d}^{\dagger} \mathbf{Y}_{d}-2 \mathbf{Y}_{d}^{\dagger} \mathbf{Y}_{d} \mathbf{Y}_{u}^{\dagger} \mathbf{Y}_{u} \\
& +\left[16 g_{3}^{2}+\frac{4}{5} g_{1}^{2}\right] \operatorname{Tr}\left(\mathbf{Y}_{u} \mathbf{Y}_{u}^{\dagger}\right)+\left[6 g_{2}^{2}+\frac{2}{5} g_{1}^{2}\right] \mathbf{Y}_{u}^{\dagger} \mathbf{Y}_{u}+\frac{2}{5} g_{1}^{2} \mathbf{Y}_{d}^{\dagger} \mathbf{Y}_{d} \\
& \left.-\frac{16}{9} g_{3}^{4}+8 g_{3}^{2} g_{2}^{2}+\frac{136}{45} g_{3}^{2} g_{1}^{2}+\frac{15}{2} g_{2}^{4}+g_{2}^{2} g_{1}^{2}+\frac{2743}{450} g_{1}^{4}\right\} \\
& \beta_{\mathbf{Y}_{d}}^{(1)}=\mathbf{Y}_{d}\left\{\operatorname{Tr}\left(3 \mathbf{Y}_{d} \mathbf{Y}_{d}^{\dagger}+\mathbf{Y}_{e} \mathbf{Y}_{e}^{\dagger}\right)+3 \mathbf{Y}_{d}^{\dagger} \mathbf{Y}_{d}+\mathbf{Y}_{u}^{\dagger} \mathbf{Y}_{u}-\frac{16}{3} g_{3}^{2}-3 g_{2}^{2}-\frac{7}{15} g_{1}^{2}\right\} \\
& \beta_{\mathbf{Y}_{d}}^{(2)}=\mathbf{Y}_{d}\left\{-3 \operatorname{Tr}\left(3 \mathbf{Y}_{d} \mathbf{Y}_{d}^{\dagger} \mathbf{Y}_{d} \mathbf{Y}_{d}^{\dagger}+\mathbf{Y}_{u} \mathbf{Y}_{d}^{\dagger} \mathbf{Y}_{d} \mathbf{Y}_{u}^{\dagger}+\mathbf{Y}_{e} \mathbf{Y}_{e}^{\dagger} \mathbf{Y}_{e} \mathbf{Y}_{e}^{\dagger}\right)\right. \\
& -3 \mathbf{Y}_{u}^{\dagger} \mathbf{Y}_{u} \operatorname{Tr}\left(\mathbf{Y}_{u} \mathbf{Y}_{u}^{\dagger}\right)-3 \mathbf{Y}_{d}^{\dagger} \mathbf{Y}_{d} \operatorname{Tr}\left(3 \mathbf{Y}_{d} \mathbf{Y}_{d}^{\dagger}+\mathbf{Y}_{e} \mathbf{Y}_{e}^{\dagger}\right)-4 \mathbf{Y}_{d}^{\dagger} \mathbf{Y}_{d} \mathbf{Y}_{d}^{\dagger} \mathbf{Y}_{d} \\
& -2 \mathbf{Y}_{u}^{\dagger} \mathbf{Y}_{u} \mathbf{Y}_{u}^{\dagger} \mathbf{Y}_{u}-2 \mathbf{Y}_{u}^{\dagger} \mathbf{Y}_{u} \mathbf{Y}_{d}^{\dagger} \mathbf{Y}_{d}+\left[16 g_{3}^{2}-\frac{2}{5} g_{1}^{2}\right] \operatorname{Tr}\left(\mathbf{Y}_{d} \mathbf{Y}_{d}^{\dagger}\right) \\
& +\frac{6}{5} g_{1}^{2} \operatorname{Tr}\left(\mathbf{Y}_{e} \mathbf{Y}_{e}^{\dagger}\right)+\frac{4}{5} g_{1}^{2} \mathbf{Y}_{u}^{\dagger} \mathbf{Y}_{u}+\left[6 g_{2}^{2}+\frac{4}{5} g_{1}^{2}\right] \mathbf{Y}_{d}^{\dagger} \mathbf{Y}_{d} \\
& \left.-\frac{16}{9} g_{3}^{4}+8 g_{3}^{2} g_{2}^{2}+\frac{8}{9} g_{3}^{2} g_{1}^{2}+\frac{15}{2} g_{2}^{4}+g_{2}^{2} g_{1}^{2}+\frac{287}{90} g_{1}^{4}\right\}
\end{aligned}
$$

$$
\begin{aligned}
\beta_{\mathbf{Y}_{e}}^{(1)}=\mathbf{Y}_{e}\{ & \left.\operatorname{Tr}\left(3 \mathbf{Y}_{d} \mathbf{Y}_{d}^{\dagger}+\mathbf{Y}_{e} \mathbf{Y}_{e}^{\dagger}\right)+3 \mathbf{Y}_{e}^{\dagger} \mathbf{Y}_{e}-3 g_{2}^{2}-\frac{9}{5} g_{1}^{2}\right\} \\
\beta_{\mathbf{Y}_{e}}^{(2)}=\mathbf{Y}_{e}\{ & -3 \operatorname{Tr}\left(3 \mathbf{Y}_{d} \mathbf{Y}_{d}^{\dagger} \mathbf{Y}_{d} \mathbf{Y}_{d}^{\dagger}+\mathbf{Y}_{u} \mathbf{Y}_{d}^{\dagger} \mathbf{Y}_{d} \mathbf{Y}_{u}^{\dagger}+\mathbf{Y}_{e} \mathbf{Y}_{e}^{\dagger} \mathbf{Y}_{e} \mathbf{Y}_{e}^{\dagger}\right) \\
& -3 \mathbf{Y}_{e}^{\dagger} \mathbf{Y}_{e} \operatorname{Tr}\left(3 \mathbf{Y}_{d} \mathbf{Y}_{d}^{\dagger}+\mathbf{Y}_{e} \mathbf{Y}_{e}^{\dagger}\right)-4 \mathbf{Y}_{e}^{\dagger} \mathbf{Y}_{e} \mathbf{Y}_{e}^{\dagger} \mathbf{Y}_{e}+\left[16 g_{3}^{2}-\frac{2}{5} g_{1}^{2}\right] \operatorname{Tr}\left(\mathbf{Y}_{d} \mathbf{Y}_{d}^{\dagger}\right) \\
& \left.+\frac{6}{5} g_{1}^{2} \operatorname{Tr}\left(\mathbf{Y}_{e} \mathbf{Y}_{e}^{\dagger}\right)+6 g_{2}^{2} \mathbf{Y}_{e}^{\dagger} \mathbf{Y}_{e}+\frac{15}{2} g_{2}^{4}+\frac{9}{5} g_{2}^{2} g_{1}^{2}+\frac{27}{2} g_{1}^{4}\right\}
\end{aligned}
$$

Of course, the $\beta$-functions for $\mathbf{Y}_{u, d, e}$ are $3 \times 3$ matrices in family space. 
The above results for the MSSM have all appeared before. Now we apply our results of Sections II and III to arrive at the two-loop beta functions for the soft-breaking trilinear scalar couplings:

$$
\begin{aligned}
& \frac{d}{d t} \mathbf{h}_{u, d, e}=\frac{1}{16 \pi^{2}} \beta_{\mathbf{h}_{u, d, e}}^{(1)}+\frac{1}{\left(16 \pi^{2}\right)^{2}} \beta_{\mathbf{h}_{u, d, e}}^{(2)} . \\
& \beta_{\mathbf{h}_{u}}^{(1)}=\mathbf{h}_{u}\left\{3 \operatorname{Tr}\left(\mathbf{Y}_{u} \mathbf{Y}_{u}^{\dagger}\right)+5 \mathbf{Y}_{u}^{\dagger} \mathbf{Y}_{u}+\mathbf{Y}_{d}^{\dagger} \mathbf{Y}_{d}-\frac{16}{3} g_{3}^{2}-3 g_{2}^{2}-\frac{13}{15} g_{1}^{2}\right\} \\
& +\mathbf{Y}_{u}\left\{6 \operatorname{Tr}\left(\mathbf{h}_{u} \mathbf{Y}_{u}^{\dagger}\right)+4 \mathbf{Y}_{u}^{\dagger} \mathbf{h}_{u}+2 \mathbf{Y}_{d}^{\dagger} \mathbf{h}_{d}+\frac{32}{3} g_{3}^{2} M_{3}+6 g_{2}^{2} M_{2}+\frac{26}{15} g_{1}^{2} M_{1}\right\} \\
& \beta_{\mathbf{h}_{u}}^{(2)}=\mathbf{h}_{u}\left\{-3 \operatorname{Tr}\left(3 \mathbf{Y}_{u} \mathbf{Y}_{u}^{\dagger} \mathbf{Y}_{u} \mathbf{Y}_{u}^{\dagger}+\mathbf{Y}_{u} \mathbf{Y}_{d}^{\dagger} \mathbf{Y}_{d} \mathbf{Y}_{u}^{\dagger}\right)-\mathbf{Y}_{d}^{\dagger} \mathbf{Y}_{d} \operatorname{Tr}\left(3 \mathbf{Y}_{d} \mathbf{Y}_{d}^{\dagger}+\mathbf{Y}_{e} \mathbf{Y}_{e}^{\dagger}\right)\right. \\
& -15 \mathbf{Y}_{u}^{\dagger} \mathbf{Y}_{u} \operatorname{Tr}\left(\mathbf{Y}_{u} \mathbf{Y}_{u}^{\dagger}\right)-6 \mathbf{Y}_{u}^{\dagger} \mathbf{Y}_{u} \mathbf{Y}_{u}^{\dagger} \mathbf{Y}_{u}-2 \mathbf{Y}_{d}^{\dagger} \mathbf{Y}_{d} \mathbf{Y}_{d}^{\dagger} \mathbf{Y}_{d}-4 \mathbf{Y}_{d}^{\dagger} \mathbf{Y}_{d} \mathbf{Y}_{u}^{\dagger} \mathbf{Y}_{u} \\
& +\left[16 g_{3}^{2}+\frac{4}{5} g_{1}^{2}\right] \operatorname{Tr}\left(\mathbf{Y}_{u} \mathbf{Y}_{u}^{\dagger}\right)+12 g_{2}^{2} \mathbf{Y}_{u}^{\dagger} \mathbf{Y}_{u}+\frac{2}{5} g_{1}^{2} \mathbf{Y}_{d}^{\dagger} \mathbf{Y}_{d} \\
& \left.-\frac{16}{9} g_{3}^{4}+8 g_{3}^{2} g_{2}^{2}+\frac{136}{45} g_{3}^{2} g_{1}^{2}+\frac{15}{2} g_{2}^{4}+g_{2}^{2} g_{1}^{2}+\frac{2743}{450} g_{1}^{4}\right\} \\
& +\mathbf{Y}_{u}\left\{-6 \operatorname{Tr}\left(6 \mathbf{h}_{u} \mathbf{Y}_{u}^{\dagger} \mathbf{Y}_{u} \mathbf{Y}_{u}^{\dagger}+\mathbf{h}_{u} \mathbf{Y}_{d}^{\dagger} \mathbf{Y}_{d} \mathbf{Y}_{u}^{\dagger}+\mathbf{h}_{d} \mathbf{Y}_{u}^{\dagger} \mathbf{Y}_{u} \mathbf{Y}_{d}^{\dagger}\right)\right. \\
& -18 \mathbf{Y}_{u}^{\dagger} \mathbf{Y}_{u} \operatorname{Tr}\left(\mathbf{h}_{u} \mathbf{Y}_{u}^{\dagger}\right)-\mathbf{Y}_{d}^{\dagger} \mathbf{Y}_{d} \operatorname{Tr}\left(6 \mathbf{h}_{d} \mathbf{Y}_{d}^{\dagger}+2 \mathbf{h}_{e} \mathbf{Y}_{e}^{\dagger}\right)-12 \mathbf{Y}_{u}^{\dagger} \mathbf{h}_{u} \operatorname{Tr}\left(\mathbf{Y}_{u} \mathbf{Y}_{u}^{\dagger}\right) \\
& -\mathbf{Y}_{d}^{\dagger} \mathbf{h}_{d} \operatorname{Tr}\left(6 \mathbf{Y}_{d} \mathbf{Y}_{d}^{\dagger}+2 \mathbf{Y}_{e} \mathbf{Y}_{e}^{\dagger}\right)-6 \mathbf{Y}_{u}^{\dagger} \mathbf{Y}_{u} \mathbf{Y}_{u}^{\dagger} \mathbf{h}_{u}-8 \mathbf{Y}_{u}^{\dagger} \mathbf{h}_{u} \mathbf{Y}_{u}^{\dagger} \mathbf{Y}_{u} \\
& -4 \mathbf{Y}_{d}^{\dagger} \mathbf{Y}_{d} \mathbf{Y}_{d}^{\dagger} \mathbf{h}_{d}-4 \mathbf{Y}_{d}^{\dagger} \mathbf{h}_{d} \mathbf{Y}_{d}^{\dagger} \mathbf{Y}_{d}-2 \mathbf{Y}_{d}^{\dagger} \mathbf{Y}_{d} \mathbf{Y}_{u}^{\dagger} \mathbf{h}_{u}-4 \mathbf{Y}_{d}^{\dagger} \mathbf{h}_{d} \mathbf{Y}_{u}^{\dagger} \mathbf{Y}_{u} \\
& +\left[32 g_{3}^{2}+\frac{8}{5} g_{1}^{2}\right] \operatorname{Tr}\left(\mathbf{h}_{u} \mathbf{Y}_{u}^{\dagger}\right)+\left[6 g_{2}^{2}+\frac{6}{5} g_{1}^{2}\right] \mathbf{Y}_{u}^{\dagger} \mathbf{h}_{u}+\frac{4}{5} g_{1}^{2} \mathbf{Y}_{d}^{\dagger} \mathbf{h}_{d} \\
& -\left[32 g_{3}^{2} M_{3}+\frac{8}{5} g_{1}^{2} M_{1}\right] \operatorname{Tr}\left(\mathbf{Y}_{u} \mathbf{Y}_{u}^{\dagger}\right)-\left[12 g_{2}^{2} M_{2}+\frac{4}{5} g_{1}^{2} M_{1}\right] \mathbf{Y}_{u}^{\dagger} \mathbf{Y}_{u} \\
& -\frac{4}{5} g_{1}^{2} M_{1} \mathbf{Y}_{d}^{\dagger} \mathbf{Y}_{d}+\frac{64}{9} g_{3}^{4} M_{3}-16 g_{3}^{2} g_{2}^{2}\left(M_{3}+M_{2}\right)-\frac{272}{45} g_{3}^{2} g_{1}^{2}\left(M_{3}+M_{1}\right) \\
& \left.-30 g_{2}^{4} M_{2}-2 g_{2}^{2} g_{1}^{2}\left(M_{2}+M_{1}\right)-\frac{5486}{225} g_{1}^{4} M_{1}\right\} \\
& \beta_{\mathbf{h}_{d}}^{(1)}=\mathbf{h}_{d}\left\{\operatorname{Tr}\left(3 \mathbf{Y}_{d} \mathbf{Y}_{d}^{\dagger}+\mathbf{Y}_{e} \mathbf{Y}_{e}^{\dagger}\right)+5 \mathbf{Y}_{d}^{\dagger} \mathbf{Y}_{d}+\mathbf{Y}_{u}^{\dagger} \mathbf{Y}_{u}-\frac{16}{3} g_{3}^{2}-3 g_{2}^{2}-\frac{7}{15} g_{1}^{2}\right\} \\
& +\mathbf{Y}_{d}\left\{\operatorname{Tr}\left(6 \mathbf{h}_{d} \mathbf{Y}_{d}^{\dagger}+2 \mathbf{h}_{e} \mathbf{Y}_{e}^{\dagger}\right)+4 \mathbf{Y}_{d}^{\dagger} \mathbf{h}_{d}+2 \mathbf{Y}_{u}^{\dagger} \mathbf{h}_{u}+\frac{32}{3} g_{3}^{2} M_{3}+6 g_{2}^{2} M_{2}+\frac{14}{15} g_{1}^{2} M_{1}\right\} \\
& \beta_{\mathbf{h}_{d}}^{(2)}=\mathbf{h}_{d}\left\{-3 \operatorname{Tr}\left(3 \mathbf{Y}_{d} \mathbf{Y}_{d}^{\dagger} \mathbf{Y}_{d} \mathbf{Y}_{d}^{\dagger}+\mathbf{Y}_{u} \mathbf{Y}_{d}^{\dagger} \mathbf{Y}_{d} \mathbf{Y}_{u}^{\dagger}+\mathbf{Y}_{e} \mathbf{Y}_{e}^{\dagger} \mathbf{Y}_{e} \mathbf{Y}_{e}^{\dagger}\right)\right.
\end{aligned}
$$




$$
\begin{aligned}
& -3 \mathbf{Y}_{u}^{\dagger} \mathbf{Y}_{u} \operatorname{Tr}\left(\mathbf{Y}_{u} \mathbf{Y}_{u}^{\dagger}\right)-5 \mathbf{Y}_{d}^{\dagger} \mathbf{Y}_{d} \operatorname{Tr}\left(3 \mathbf{Y}_{d} \mathbf{Y}_{d}^{\dagger}+\mathbf{Y}_{e} \mathbf{Y}_{e}^{\dagger}\right)-6 \mathbf{Y}_{d}^{\dagger} \mathbf{Y}_{d} \mathbf{Y}_{d}^{\dagger} \mathbf{Y}_{d} \\
& -2 \mathbf{Y}_{u}^{\dagger} \mathbf{Y}_{u} \mathbf{Y}_{u}^{\dagger} \mathbf{Y}_{u}-4 \mathbf{Y}_{u}^{\dagger} \mathbf{Y}_{u} \mathbf{Y}_{d}^{\dagger} \mathbf{Y}_{d}+\left[16 g_{3}^{2}-\frac{2}{5} g_{1}^{2}\right] \operatorname{Tr}\left(\mathbf{Y}_{d} \mathbf{Y}_{d}^{\dagger}\right) \\
& +\frac{6}{5} g_{1}^{2} \operatorname{Tr}\left(\mathbf{Y}_{e} \mathbf{Y}_{e}^{\dagger}\right)+\frac{4}{5} g_{1}^{2} \mathbf{Y}_{u}^{\dagger} \mathbf{Y}_{u}+\left[12 g_{2}^{2}+\frac{6}{5} g_{1}^{2}\right] \mathbf{Y}_{d}^{\dagger} \mathbf{Y}_{d} \\
& \left.-\frac{16}{9} g_{3}^{4}+8 g_{3}^{2} g_{2}^{2}+\frac{8}{9} g_{3}^{2} g_{1}^{2}+\frac{15}{2} g_{2}^{4}+g_{2}^{2} g_{1}^{2}+\frac{287}{90} g_{1}^{4}\right\} \\
+\mathbf{Y}_{d}\{ & -6 \operatorname{Tr}\left(6 \mathbf{h}_{d} \mathbf{Y}_{d}^{\dagger} \mathbf{Y}_{d} \mathbf{Y}_{d}^{\dagger}+\mathbf{h}_{u} \mathbf{Y}_{d}^{\dagger} \mathbf{Y}_{d} \mathbf{Y}_{u}^{\dagger}+\mathbf{h}_{d} \mathbf{Y}_{u}^{\dagger} \mathbf{Y}_{u} \mathbf{Y}_{d}^{\dagger}+2 \mathbf{h}_{e} \mathbf{Y}_{e}^{\dagger} \mathbf{Y}_{e} \mathbf{Y}_{e}^{\dagger}\right) \\
& -6 \mathbf{Y}_{u}^{\dagger} \mathbf{Y}_{u} \operatorname{Tr}\left(\mathbf{h}_{u} \mathbf{Y}_{u}^{\dagger}\right)-6 \mathbf{Y}_{d}^{\dagger} \mathbf{Y}_{d} \operatorname{Tr}\left(3 \mathbf{h}_{d} \mathbf{Y}_{d}^{\dagger}+\mathbf{h}_{e} \mathbf{Y}_{e}^{\dagger}\right) \\
& -6 \mathbf{Y}_{u}^{\dagger} \mathbf{h}_{u} \operatorname{Tr}\left(\mathbf{Y}_{u} \mathbf{Y}_{u}^{\dagger}\right)-4 \mathbf{Y}_{d}^{\dagger} \mathbf{h}_{d} \operatorname{Tr}\left(3 \mathbf{Y}_{d} \mathbf{Y}_{d}^{\dagger}+\mathbf{Y}_{e} \mathbf{Y}_{e}^{\dagger}\right)-6 \mathbf{Y}_{d}^{\dagger} \mathbf{Y}_{d} \mathbf{Y}_{d}^{\dagger} \mathbf{h}_{d} \\
& -8 \mathbf{Y}_{d}^{\dagger} \mathbf{h}_{d} \mathbf{Y}_{d}^{\dagger} \mathbf{Y}_{d}-4 \mathbf{Y}_{u}^{\dagger} \mathbf{h}_{u} \mathbf{Y}_{u}^{\dagger} \mathbf{Y}_{u}-4 \mathbf{Y}_{u}^{\dagger} \mathbf{Y}_{u} \mathbf{Y}_{u}^{\dagger} \mathbf{h}_{u}-4 \mathbf{Y}_{u}^{\dagger} \mathbf{h}_{u} \mathbf{Y}_{d}^{\dagger} \mathbf{Y}_{d} \\
& -2 \mathbf{Y}_{u}^{\dagger} \mathbf{Y}_{u} \mathbf{Y}_{d}^{\dagger} \mathbf{h}_{d}+\left[32 g_{3}^{2}-\frac{4}{5} g_{1}^{2}\right] \operatorname{Tr}\left(\mathbf{h}_{d} \mathbf{Y}_{d}^{\dagger}\right)+\frac{12}{5} g_{1}^{2} \operatorname{Tr}\left(\mathbf{h}_{e} \mathbf{Y}_{e}^{\dagger}\right)+\frac{8}{5} g_{1}^{2} \mathbf{Y}_{u}^{\dagger} \mathbf{h}_{u} \\
& +\left[6 g_{2}^{2}+\frac{6}{5} g_{1}^{2}\right] \mathbf{Y}_{d}^{\dagger} \mathbf{h}_{d}-\left[32 g_{3}^{2} M_{3}-\frac{4}{5} g_{1}^{2} M_{1}\right] \operatorname{Tr}\left(\mathbf{Y}_{d} \mathbf{Y}_{d}^{\dagger}\right)-\frac{12}{5} g_{1}^{2} M_{1} \operatorname{Tr}\left(\mathbf{Y}_{e} \mathbf{Y}_{e}^{\dagger}\right) \\
& -\left[12 g_{2}^{2} M_{2}+\frac{8}{5} g_{1}^{2} M_{1}\right] \mathbf{Y}_{d}^{\dagger} \mathbf{Y}_{d}-\frac{8}{5} g_{1}^{2} M_{1} \mathbf{Y}_{u}^{\dagger} \mathbf{Y}_{u}+\frac{64}{9} g_{3}^{4} M_{3}-16 g_{3}^{2} g_{2}^{2}\left(M_{3}+M_{2}\right) \\
& \left.-\frac{16}{9} g_{3}^{2} g_{1}^{2}\left(M_{3}+M_{1}\right)-30 g_{2}^{4} M_{2}-2 g_{2}^{2} g_{1}^{2}\left(M_{2}+M_{1}\right)-\frac{574}{45} g_{1}^{4} M_{1}\right\}
\end{aligned}
$$

$$
\begin{aligned}
\beta_{\mathbf{h}_{e}}^{(1)} & =\mathbf{h}_{e}\left\{\operatorname{Tr}\left(3 \mathbf{Y}_{d} \mathbf{Y}_{d}^{\dagger}+\mathbf{Y}_{e} \mathbf{Y}_{e}^{\dagger}\right)+5 \mathbf{Y}_{e}^{\dagger} \mathbf{Y}_{e}-3 g_{2}^{2}-\frac{9}{5} g_{1}^{2}\right\} \\
& +\mathbf{Y}_{e}\left\{\operatorname{Tr}\left(6 \mathbf{h}_{d} \mathbf{Y}_{d}^{\dagger}+2 \mathbf{h}_{e} \mathbf{Y}_{e}^{\dagger}\right)+4 \mathbf{Y}_{e}^{\dagger} \mathbf{h}_{e}+6 g_{2}^{2} M_{2}+\frac{18}{5} g_{1}^{2} M_{1}\right\}
\end{aligned}
$$

$$
\begin{aligned}
\beta_{\mathbf{h}_{e}}^{(2)}=\mathbf{h}_{e}\{ & -3 \operatorname{Tr}\left(3 \mathbf{Y}_{d} \mathbf{Y}_{d}^{\dagger} \mathbf{Y}_{d} \mathbf{Y}_{d}^{\dagger}+\mathbf{Y}_{u} \mathbf{Y}_{d}^{\dagger} \mathbf{Y}_{d} \mathbf{Y}_{u}^{\dagger}+\mathbf{Y}_{e} \mathbf{Y}_{e}^{\dagger} \mathbf{Y}_{e} \mathbf{Y}_{e}^{\dagger}\right) \\
& -5 \mathbf{Y}_{e}^{\dagger} \mathbf{Y}_{e} \operatorname{Tr}\left(3 \mathbf{Y}_{d} \mathbf{Y}_{d}^{\dagger}+\mathbf{Y}_{e} \mathbf{Y}_{e}^{\dagger}\right)-6 \mathbf{Y}_{e}^{\dagger} \mathbf{Y}_{e} \mathbf{Y}_{e}^{\dagger} \mathbf{Y}_{e}+\left[16 g_{3}^{2}-\frac{2}{5} g_{1}^{2}\right] \operatorname{Tr}\left(\mathbf{Y}_{d} \mathbf{Y}_{d}^{\dagger}\right) \\
& \left.+\frac{6}{5} g_{1}^{2} \operatorname{Tr}\left(\mathbf{Y}_{e} \mathbf{Y}_{e}^{\dagger}\right)+\left[12 g_{2}^{2}-\frac{6}{5} g_{1}^{2}\right] \mathbf{Y}_{e}^{\dagger} \mathbf{Y}_{e}+\frac{15}{2} g_{2}^{4}+\frac{9}{5} g_{2}^{2} g_{1}^{2}+\frac{27}{2} g_{1}^{4}\right\} \\
+\mathbf{Y}_{e}\{ & -6 \operatorname{Tr}\left(6 \mathbf{h}_{d} \mathbf{Y}_{d}^{\dagger} \mathbf{Y}_{d} \mathbf{Y}_{d}^{\dagger}+\mathbf{h}_{u} \mathbf{Y}_{d}^{\dagger} \mathbf{Y}_{d} \mathbf{Y}_{u}^{\dagger}+\mathbf{h}_{d} \mathbf{Y}_{u}^{\dagger} \mathbf{Y}_{u} \mathbf{Y}_{d}^{\dagger}+2 \mathbf{h}_{e} \mathbf{Y}_{e}^{\dagger} \mathbf{Y}_{e} \mathbf{Y}_{e}^{\dagger}\right) \\
& -4 \mathbf{Y}_{e}^{\dagger} \mathbf{h}_{e} \operatorname{Tr}\left(3 \mathbf{Y}_{d} \mathbf{Y}_{d}^{\dagger}+\mathbf{Y}_{e} \mathbf{Y}_{e}^{\dagger}\right)-6 \mathbf{Y}_{e}^{\dagger} \mathbf{Y}_{e} \operatorname{Tr}\left(3 \mathbf{h}_{d} \mathbf{Y}_{d}^{\dagger}+\mathbf{h}_{e} \mathbf{Y}_{e}^{\dagger}\right) \\
& -6 \mathbf{Y}_{e}^{\dagger} \mathbf{Y}_{e} \mathbf{Y}_{e}^{\dagger} \mathbf{h}_{e}-8 \mathbf{Y}_{e}^{\dagger} \mathbf{h}_{e} \mathbf{Y}_{e}^{\dagger} \mathbf{Y}_{e} \\
& +\left[32 g_{3}^{2}-\frac{4}{5} g_{1}^{2}\right] \operatorname{Tr}\left(\mathbf{h}_{d} \mathbf{Y}_{d}^{\dagger}\right)+\frac{12}{5} g_{1}^{2} \operatorname{Tr}\left(\mathbf{h}_{e} \mathbf{Y}_{e}^{\dagger}\right)+\left[6 g_{2}^{2}+\frac{6}{5} g_{1}^{2}\right] \mathbf{Y}_{e}^{\dagger} \mathbf{h}_{e} \\
& -\left[32 g_{3}^{2} M_{3}-\frac{4}{5} g_{1}^{2} M_{1}\right] \operatorname{Tr}\left(\mathbf{Y}_{d} \mathbf{Y}_{d}^{\dagger}\right)-\frac{12}{5} g_{1}^{2} M_{1} \operatorname{Tr}\left(\mathbf{Y}_{e} \mathbf{Y}_{e}^{\dagger}\right)-12 g_{2}^{2} M_{2} \mathbf{Y}_{e}^{\dagger} \mathbf{Y}_{e}
\end{aligned}
$$




$$
\left.-30 g_{2}^{4} M_{2}-\frac{18}{5} g_{2}^{2} g_{1}^{2}\left(M_{1}+M_{2}\right)-54 g_{1}^{4} M_{1}\right\}
$$

These are again $3 \times 3$ matrices in family space. One should note that there is no universally agreed-upon convention for the sign of the gaugino masses in these equations, because of the freedom to rotate the phases of the gaugino mass terms in the Lagrangian.

The MSSM also contains one scalar (mass) ${ }^{2}$ of the type $b^{i j}$, which from (2.15)-(2.17) satisfies the two-loop renormalization group equation:

$$
\begin{aligned}
& \frac{d}{d t} B=\frac{1}{16 \pi^{2}} \beta_{B}^{(1)}+\frac{1}{\left(16 \pi^{2}\right)^{2}} \beta_{B}^{(2)} \\
& \beta_{B}^{(1)}=B\left\{\operatorname{Tr}\left(3 \mathbf{Y}_{u} \mathbf{Y}_{u}^{\dagger}+3 \mathbf{Y}_{d} \mathbf{Y}_{d}^{\dagger}+\mathbf{Y}_{e} \mathbf{Y}_{e}^{\dagger}\right)-3 g_{2}^{2}-\frac{3}{5} g_{1}^{2}\right\} \\
& +\mu\left\{\operatorname{Tr}\left(6 \mathbf{h}_{u} \mathbf{Y}_{u}^{\dagger}+6 \mathbf{h}_{d} \mathbf{Y}_{d}^{\dagger}+2 \mathbf{h}_{e} \mathbf{Y}_{e}^{\dagger}\right)+6 g_{2}^{2} M_{2}+\frac{6}{5} g_{1}^{2} M_{1}\right\} \\
& \beta_{B}^{(2)}=B\left\{-3 \operatorname{Tr}\left(3 \mathbf{Y}_{u} \mathbf{Y}_{u}^{\dagger} \mathbf{Y}_{u} \mathbf{Y}_{u}^{\dagger}+3 \mathbf{Y}_{d} \mathbf{Y}_{d}^{\dagger} \mathbf{Y}_{d} \mathbf{Y}_{d}^{\dagger}+2 \mathbf{Y}_{u} \mathbf{Y}_{d}^{\dagger} \mathbf{Y}_{d} \mathbf{Y}_{u}^{\dagger}+\mathbf{Y}_{e} \mathbf{Y}_{e}^{\dagger} \mathbf{Y}_{e} \mathbf{Y}_{e}^{\dagger}\right)\right. \\
& +\left[16 g_{3}^{2}+\frac{4}{5} g_{1}^{2}\right] \operatorname{Tr}\left(\mathbf{Y}_{u} \mathbf{Y}_{u}^{\dagger}\right)+\left[16 g_{3}^{2}-\frac{2}{5} g_{1}^{2}\right] \operatorname{Tr}\left(\mathbf{Y}_{d} \mathbf{Y}_{d}^{\dagger}\right)+\frac{6}{5} g_{1}^{2} \operatorname{Tr}\left(\mathbf{Y}_{e} \mathbf{Y}_{e}^{\dagger}\right) \\
& \left.+\frac{15}{2} g_{2}^{4}+\frac{9}{5} g_{1}^{2} g_{2}^{2}+\frac{207}{50} g_{1}^{4}\right\} \\
& +\mu\left\{-12 \operatorname{Tr}\left(3 \mathbf{h}_{u} \mathbf{Y}_{u}^{\dagger} \mathbf{Y}_{u} \mathbf{Y}_{u}^{\dagger}+3 \mathbf{h}_{d} \mathbf{Y}_{d}^{\dagger} \mathbf{Y}_{d} \mathbf{Y}_{d}^{\dagger}+\mathbf{h}_{u} \mathbf{Y}_{d}^{\dagger} \mathbf{Y}_{d} \mathbf{Y}_{u}^{\dagger}+\mathbf{h}_{d} \mathbf{Y}_{u}^{\dagger} \mathbf{Y}_{u} \mathbf{Y}_{d}^{\dagger}+\mathbf{h}_{e} \mathbf{Y}_{e}^{\dagger} \mathbf{Y}_{e} \mathbf{Y}_{e}^{\dagger}\right)\right. \\
& +\left[32 g_{3}^{2}+\frac{8}{5} g_{1}^{2}\right] \operatorname{Tr}\left(\mathbf{h}_{u} \mathbf{Y}_{u}^{\dagger}\right)+\left[32 g_{3}^{2}-\frac{4}{5} g_{1}^{2}\right] \operatorname{Tr}\left(\mathbf{h}_{d} \mathbf{Y}_{d}^{\dagger}\right)+\frac{12}{5} g_{1}^{2} \operatorname{Tr}\left(\mathbf{h}_{e} \mathbf{Y}_{e}^{\dagger}\right) \\
& -\left[32 g_{3}^{2} M_{3}+\frac{8}{5} g_{1}^{2} M_{1}\right] \operatorname{Tr}\left(\mathbf{Y}_{u} \mathbf{Y}_{u}^{\dagger}\right)-\left[32 g_{3}^{2} M_{3}-\frac{4}{5} g_{1}^{2} M_{1}\right] \operatorname{Tr}\left(\mathbf{Y}_{d} \mathbf{Y}_{d}^{\dagger}\right) \\
& \left.-\frac{12}{5} g_{1}^{2} M_{1} \operatorname{Tr}\left(\mathbf{Y}_{e} \mathbf{Y}_{e}^{\dagger}\right)-30 g_{2}^{4} M_{2}-\frac{18}{5} g_{1}^{2} g_{2}^{2}\left(M_{1}+M_{2}\right)-\frac{414}{25} g_{1}^{4} M_{1}\right\} \text {. }
\end{aligned}
$$

Finally, we turn to the $\beta$-functions for the scalar (mass) $)^{2}$ terms of the $\left(m^{2}\right)_{i}^{j}$ type in the MSSM. It is convenient to define the quantities

$$
\mathcal{S}=m_{H_{u}}^{2}-m_{H_{d}}^{2}+\operatorname{Tr}\left[\mathbf{m}_{Q}^{2}-\mathbf{m}_{L}^{2}-2 \mathbf{m}_{u}^{2}+\mathbf{m}_{d}^{2}+\mathbf{m}_{e}^{2}\right]
$$

and

$$
\mathcal{S}^{\prime}=\operatorname{Tr}\left[-\left(3 m_{H_{u}}^{2}+\mathbf{m}_{Q}^{2}\right) \mathbf{Y}_{u}^{\dagger} \mathbf{Y}_{u}+4 \mathbf{Y}_{u}^{\dagger} \mathbf{m}_{u}^{2} \mathbf{Y}_{u}+\left(3 m_{H_{d}}^{2}-\mathbf{m}_{Q}^{2}\right) \mathbf{Y}_{d}^{\dagger} \mathbf{Y}_{d}-2 \mathbf{Y}_{d}^{\dagger} \mathbf{m}_{d}^{2} \mathbf{Y}_{d}\right.
$$




$$
\begin{gathered}
\left.+\left(m_{H_{d}}^{2}+\mathbf{m}_{L}^{2}\right) \mathbf{Y}_{e}^{\dagger} \mathbf{Y}_{e}-2 \mathbf{Y}_{e}^{\dagger} \mathbf{m}_{e}^{2} \mathbf{Y}_{e}\right] \\
+\left[\frac{3}{2} g_{2}^{2}+\frac{3}{10} g_{1}^{2}\right]\left\{m_{H_{u}}^{2}-m_{H_{d}}^{2}-\operatorname{Tr}\left(\mathbf{m}_{L}^{2}\right)\right\}+\left[\frac{8}{3} g_{3}^{2}+\frac{3}{2} g_{2}^{2}+\frac{1}{30} g_{1}^{2}\right] \operatorname{Tr}\left(\mathbf{m}_{Q}^{2}\right) \\
-\left[\frac{16}{3} g_{3}^{2}+\frac{16}{15} g_{1}^{2}\right] \operatorname{Tr}\left(\mathbf{m}_{u}^{2}\right)+\left[\frac{8}{3} g_{3}^{2}+\frac{2}{15} g_{1}^{2}\right] \operatorname{Tr}\left(\mathbf{m}_{d}^{2}\right)+\frac{6}{5} g_{1}^{2} \operatorname{Tr}\left(\mathbf{m}_{e}^{2}\right)
\end{gathered}
$$

and

$$
\begin{aligned}
& \sigma_{1}=\frac{1}{5} g_{1}^{2}\left\{3\left(m_{H_{u}}^{2}+m_{H_{d}}^{2}\right)+\operatorname{Tr}\left[\mathbf{m}_{Q}^{2}+3 \mathbf{m}_{L}^{2}+8 \mathbf{m}_{u}^{2}+2 \mathbf{m}_{d}^{2}+6 \mathbf{m}_{e}^{2}\right]\right\} \\
& \sigma_{2}=g_{2}^{2}\left\{m_{H_{u}}^{2}+m_{H_{d}}^{2}+\operatorname{Tr}\left[3 \mathbf{m}_{Q}^{2}+\mathbf{m}_{L}^{2}\right]\right\} \\
& \sigma_{3}=g_{3}^{2} \operatorname{Tr}\left[2 \mathbf{m}_{Q}^{2}+\mathbf{m}_{u}^{2}+\mathbf{m}_{d}^{2}\right] .
\end{aligned}
$$

Then from the results of sections II and III, we obtain for each of the $\beta$-functions in the standard form

$$
\frac{d}{d t} m^{2}=\frac{1}{16 \pi^{2}} \beta_{m^{2}}^{(1)}+\frac{1}{\left(16 \pi^{2}\right)^{2}} \beta_{m^{2}}^{(2)}
$$

the following results:

$$
\begin{aligned}
\beta_{m_{H_{u}}^{2}}^{(1)}= & \operatorname{Tr}\left[\left(m_{H_{u}}^{2}+\mathbf{m}_{Q}^{2}\right) \mathbf{Y}_{u}^{\dagger} \mathbf{Y}_{u}+\mathbf{Y}_{u}^{\dagger} \mathbf{m}_{u}^{2} \mathbf{Y}_{u}+\mathbf{h}_{u}^{\dagger} \mathbf{h}_{u}\right] \\
& -6 g_{2}^{2}\left|M_{2}\right|^{2}-\frac{6}{5} g_{1}^{2}\left|M_{1}\right|^{2}+\frac{3}{5} g_{1}^{2} \mathcal{S} \\
\beta_{m_{H_{u}}^{2}}^{(2)}= & -6 \operatorname{Tr}\left[6\left(m_{H_{u}}^{2}+\mathbf{m}_{Q}^{2}\right) \mathbf{Y}_{u}^{\dagger} \mathbf{Y}_{u} \mathbf{Y}_{u}^{\dagger} \mathbf{Y}_{u}+6 \mathbf{Y}_{u}^{\dagger} \mathbf{m}_{u}^{2} \mathbf{Y}_{u} \mathbf{Y}_{u}^{\dagger} \mathbf{Y}_{u}\right. \\
& +\left(m_{H_{u}}^{2}+m_{H_{d}}^{2}+\mathbf{m}_{Q}^{2}\right) \mathbf{Y}_{u}^{\dagger} \mathbf{Y}_{u} \mathbf{Y}_{d}^{\dagger} \mathbf{Y}_{d}+\mathbf{Y}_{u}^{\dagger} \mathbf{m}_{u}^{2} \mathbf{Y}_{u} \mathbf{Y}_{d}^{\dagger} \mathbf{Y}_{d} \\
& \quad+\mathbf{Y}_{u}^{\dagger} \mathbf{Y}_{u} \mathbf{m}_{Q}^{2} \mathbf{Y}_{d}^{\dagger} \mathbf{Y}_{d}+\mathbf{Y}_{u}^{\dagger} \mathbf{Y}_{u} \mathbf{Y}_{d}^{\dagger} \mathbf{m}_{d}^{2} \mathbf{Y}_{d}+6 \mathbf{h}_{u}^{\dagger} \mathbf{h}_{u} \mathbf{Y}_{u}^{\dagger} \mathbf{Y}_{u}+6 \mathbf{h}_{u}^{\dagger} \mathbf{Y}_{u} \mathbf{Y}_{u}^{\dagger} \mathbf{h}_{u} \\
& \left.\quad+\mathbf{h}_{d}^{\dagger} \mathbf{h}_{d} \mathbf{Y}_{u}^{\dagger} \mathbf{Y}_{u}+\mathbf{Y}_{d}^{\dagger} \mathbf{Y}_{d} \mathbf{h}_{u}^{\dagger} \mathbf{h}_{u}+\mathbf{h}_{d}^{\dagger} \mathbf{Y}_{d} \mathbf{Y}_{u}^{\dagger} \mathbf{h}_{u}+\mathbf{Y}_{d}^{\dagger} \mathbf{h}_{d} \mathbf{h}_{u}^{\dagger} \mathbf{Y}_{u}\right] \\
& +\left[32 g_{3}^{2}+\frac{8}{5} g_{1}^{2}\right] \operatorname{Tr}\left[\left(m_{H_{u}}^{2}+\mathbf{m}_{Q}^{2}\right) \mathbf{Y}_{u}^{\dagger} \mathbf{Y}_{u}+\mathbf{Y}_{u}^{\dagger} \mathbf{m}_{u}^{2} \mathbf{Y}_{u}+\mathbf{h}_{u}^{\dagger} \mathbf{h}_{u}\right] \\
& +32 g_{3}^{2}\left\{2\left|M_{3}\right|^{2} \operatorname{Tr}\left[\mathbf{Y}_{u}^{\dagger} \mathbf{Y}_{u}\right]-M_{3}^{*} \operatorname{Tr}\left[\mathbf{Y}_{u}^{\dagger} \mathbf{h}_{u}\right]-M_{3} \operatorname{Tr}\left[\mathbf{h}_{u}^{\dagger} \mathbf{Y}_{u}\right]\right\} \\
& +\frac{8}{5} g_{1}^{2}\left\{2\left|M_{1}\right|^{2} \operatorname{Tr}\left[\mathbf{Y}_{u}^{\dagger} \mathbf{Y}_{u}\right]-M_{1}^{*} \operatorname{Tr}\left[\mathbf{Y}_{u}^{\dagger} \mathbf{h}_{u}\right]-M_{1} \operatorname{Tr}\left[\mathbf{h}_{u}^{\dagger} \mathbf{Y}_{u}\right]\right\}+\frac{6}{5} g_{1}^{2} \mathcal{S}^{\prime} \\
& +33 g_{2}^{4}\left|M_{2}\right|^{2}+\frac{18}{5} g_{2}^{2} g_{1}^{2}\left(\left|M_{2}\right|^{2}+\left|M_{1}\right|^{2}+\operatorname{Re}\left[M_{1} M_{2}^{*}\right]\right)+\frac{621}{25} g_{1}^{4}\left|M_{1}\right|^{2} \\
& +3 g_{2}^{2} \sigma_{2}+\frac{3}{5} g_{1}^{2} \sigma_{1}
\end{aligned}
$$




$$
\begin{aligned}
& \beta_{m_{H_{d}}^{2}}^{(1)}=\operatorname{Tr}\left[6\left(m_{H_{d}}^{2}+\mathbf{m}_{Q}^{2}\right) \mathbf{Y}_{d}^{\dagger} \mathbf{Y}_{d}+6 \mathbf{Y}_{d}^{\dagger} \mathbf{m}_{d}^{2} \mathbf{Y}_{d}+2\left(m_{H_{d}}^{2}+\mathbf{m}_{L}^{2}\right) \mathbf{Y}_{e}^{\dagger} \mathbf{Y}_{e}+2 \mathbf{Y}_{e}^{\dagger} \mathbf{m}_{e}^{2} \mathbf{Y}_{e}\right. \\
& \left.+6 \mathbf{h}_{d}^{\dagger} \mathbf{h}_{d}+2 \mathbf{h}_{e}^{\dagger} \mathbf{h}_{e}\right]-6 g_{2}^{2}\left|M_{2}\right|^{2}-\frac{6}{5} g_{1}^{2}\left|M_{1}\right|^{2}-\frac{3}{5} g_{1}^{2} \mathcal{S} \\
& \beta_{m_{H_{d}}^{2}}^{(2)}=-6 \operatorname{Tr}\left[6\left(m_{H_{d}}^{2}+\mathbf{m}_{Q}^{2}\right) \mathbf{Y}_{d}^{\dagger} \mathbf{Y}_{d} \mathbf{Y}_{d}^{\dagger} \mathbf{Y}_{d}+6 \mathbf{Y}_{d}^{\dagger} \mathbf{m}_{d}^{2} \mathbf{Y}_{d} \mathbf{Y}_{d}^{\dagger} \mathbf{Y}_{d}\right. \\
& +\left(m_{H_{u}}^{2}+m_{H_{d}}^{2}+\mathbf{m}_{Q}^{2}\right) \mathbf{Y}_{u}^{\dagger} \mathbf{Y}_{u} \mathbf{Y}_{d}^{\dagger} \mathbf{Y}_{d}+\mathbf{Y}_{u}^{\dagger} \mathbf{m}_{u}^{2} \mathbf{Y}_{u} \mathbf{Y}_{d}^{\dagger} \mathbf{Y}_{d} \\
& +\mathbf{Y}_{u}^{\dagger} \mathbf{Y}_{u} \mathbf{m}_{Q}^{2} \mathbf{Y}_{d}^{\dagger} \mathbf{Y}_{d}+\mathbf{Y}_{u}^{\dagger} \mathbf{Y}_{u} \mathbf{Y}_{d}^{\dagger} \mathbf{m}_{d}^{2} \mathbf{Y}_{d}+2\left(m_{H_{d}}^{2}+\mathbf{m}_{L}^{2}\right) \mathbf{Y}_{e}^{\dagger} \mathbf{Y}_{e} \mathbf{Y}_{e}^{\dagger} \mathbf{Y}_{e} \\
& +2 \mathbf{Y}_{e}^{\dagger} \mathbf{m}_{e}^{2} \mathbf{Y}_{e} \mathbf{Y}_{e}^{\dagger} \mathbf{Y}_{e}+6 \mathbf{h}_{d}^{\dagger} \mathbf{h}_{d} \mathbf{Y}_{d}^{\dagger} \mathbf{Y}_{d}+6 \mathbf{h}_{d}^{\dagger} \mathbf{Y}_{d} \mathbf{Y}_{d}^{\dagger} \mathbf{h}_{d}+\mathbf{h}_{u}^{\dagger} \mathbf{h}_{u} \mathbf{Y}_{d}^{\dagger} \mathbf{Y}_{d} \\
& \left.+\mathbf{Y}_{u}^{\dagger} \mathbf{Y}_{u} \mathbf{h}_{d}^{\dagger} \mathbf{h}_{d}+\mathbf{h}_{u}^{\dagger} \mathbf{Y}_{u} \mathbf{Y}_{d}^{\dagger} \mathbf{h}_{d}+\mathbf{Y}_{u}^{\dagger} \mathbf{h}_{u} \mathbf{h}_{d}^{\dagger} \mathbf{Y}_{d}+2 \mathbf{h}_{e}^{\dagger} \mathbf{h}_{e} \mathbf{Y}_{e}^{\dagger} \mathbf{Y}_{e}+2 \mathbf{h}_{e}^{\dagger} \mathbf{Y}_{e} \mathbf{Y}_{e}^{\dagger} \mathbf{h}_{e}\right] \\
& +\left[32 g_{3}^{2}-\frac{4}{5} g_{1}^{2}\right] \operatorname{Tr}\left[\left(m_{H_{d}}^{2}+\mathbf{m}_{Q}^{2}\right) \mathbf{Y}_{d}^{\dagger} \mathbf{Y}_{d}+\mathbf{Y}_{d}^{\dagger} \mathbf{m}_{d}^{2} \mathbf{Y}_{d}+\mathbf{h}_{d}^{\dagger} \mathbf{h}_{d}\right] \\
& +32 g_{3}^{2}\left\{2\left|M_{3}\right|^{2} \operatorname{Tr}\left[\mathbf{Y}_{d}^{\dagger} \mathbf{Y}_{d}\right]-M_{3}^{*} \operatorname{Tr}\left[\mathbf{Y}_{d}^{\dagger} \mathbf{h}_{d}\right]-M_{3} \operatorname{Tr}\left[\mathbf{h}_{d}^{\dagger} \mathbf{Y}_{d}\right]\right\} \\
& -\frac{4}{5} g_{1}^{2}\left\{2\left|M_{1}\right|^{2} \operatorname{Tr}\left[\mathbf{Y}_{d}^{\dagger} \mathbf{Y}_{d}\right]-M_{1}^{*} \operatorname{Tr}\left[\mathbf{Y}_{d}^{\dagger} \mathbf{h}_{d}\right]-M_{1} \operatorname{Tr}\left[\mathbf{h}_{d}^{\dagger} \mathbf{Y}_{d}\right]\right\} \\
& +\frac{12}{5} g_{1}^{2}\left\{\operatorname{Tr}\left[\left(m_{H_{d}}^{2}+\mathbf{m}_{L}^{2}\right) \mathbf{Y}_{e}^{\dagger} \mathbf{Y}_{e}+\mathbf{Y}_{e}^{\dagger} \mathbf{m}_{e}^{2} \mathbf{Y}_{e}+\mathbf{h}_{e}^{\dagger} \mathbf{h}_{e}\right]+2\left|M_{1}\right|^{2} \operatorname{Tr}\left[\mathbf{Y}_{e}^{\dagger} \mathbf{Y}_{e}\right]\right. \\
& \left.-M_{1} \operatorname{Tr}\left[\mathbf{h}_{e}^{\dagger} \mathbf{Y}_{e}\right]-M_{1}^{*} \operatorname{Tr}\left[\mathbf{Y}_{e}^{\dagger} \mathbf{h}_{e}\right]\right\}-\frac{6}{5} g_{1}^{2} \mathcal{S}^{\prime}+33 g_{2}^{4}\left|M_{2}\right|^{2} \\
& +\frac{18}{5} g_{2}^{2} g_{1}^{2}\left(\left|M_{2}\right|^{2}+\left|M_{1}\right|^{2}+\operatorname{Re}\left[M_{1} M_{2}^{*}\right]\right)+\frac{621}{25} g_{1}^{4}\left|M_{1}\right|^{2} \\
& +3 g_{2}^{2} \sigma_{2}+\frac{3}{5} g_{1}^{2} \sigma_{1}
\end{aligned}
$$

The $\beta$-functions for $\mathbf{m}_{Q}^{2}, \mathbf{m}_{L}^{2}, \mathbf{m}_{u}^{2}, \mathbf{m}_{d}^{2}, \mathbf{m}_{e}^{2}$ are of course $3 \times 3$ matrices:

$$
\begin{aligned}
\beta_{\mathbf{m}_{Q}^{2}}^{(1)}= & \left(\mathbf{m}_{Q}^{2}+2 m_{H_{u}}^{2}\right) \mathbf{Y}_{u}^{\dagger} \mathbf{Y}_{u}+\left(\mathbf{m}_{Q}^{2}+2 m_{H_{d}}^{2}\right) \mathbf{Y}_{d}^{\dagger} \mathbf{Y}_{d}+\left[\mathbf{Y}_{u}^{\dagger} \mathbf{Y}_{u}+\mathbf{Y}_{d}^{\dagger} \mathbf{Y}_{d}\right] \mathbf{m}_{Q}^{2}+2 \mathbf{Y}_{u}^{\dagger} \mathbf{m}_{u}^{2} \mathbf{Y}_{u} \\
& +2 \mathbf{Y}_{d}^{\dagger} \mathbf{m}_{d}^{2} \mathbf{Y}_{d}+2 \mathbf{h}_{u}^{\dagger} \mathbf{h}_{u}+2 \mathbf{h}_{d}^{\dagger} \mathbf{h}_{d}-\frac{32}{3} g_{3}^{2}\left|M_{3}\right|^{2}-6 g_{2}^{2}\left|M_{2}\right|^{2}-\frac{2}{15} g_{1}^{2}\left|M_{1}\right|^{2}+\frac{1}{5} g_{1}^{2} \mathcal{S}
\end{aligned}
$$

$$
\begin{aligned}
\beta_{\mathbf{m}_{Q}^{2}}^{(2)}= & -\left(2 \mathbf{m}_{Q}^{2}+8 m_{H_{u}}^{2}\right) \mathbf{Y}_{u}^{\dagger} \mathbf{Y}_{u} \mathbf{Y}_{u}^{\dagger} \mathbf{Y}_{u}-4 \mathbf{Y}_{u}^{\dagger} \mathbf{m}_{u}^{2} \mathbf{Y}_{u} \mathbf{Y}_{u}^{\dagger} \mathbf{Y}_{u}-4 \mathbf{Y}_{u}^{\dagger} \mathbf{Y}_{u} \mathbf{m}_{Q}^{2} \mathbf{Y}_{u}^{\dagger} \mathbf{Y}_{u} \\
& -4 \mathbf{Y}_{u}^{\dagger} \mathbf{Y}_{u} \mathbf{Y}_{u}^{\dagger} \mathbf{m}_{u}^{2} \mathbf{Y}_{u}-2 \mathbf{Y}_{u}^{\dagger} \mathbf{Y}_{u} \mathbf{Y}_{u}^{\dagger} \mathbf{Y}_{u} \mathbf{m}_{Q}^{2}-\left(2 \mathbf{m}_{Q}^{2}+8 m_{H_{d}}^{2}\right) \mathbf{Y}_{d}^{\dagger} \mathbf{Y}_{d} \mathbf{Y}_{d}^{\dagger} \mathbf{Y}_{d} \\
& -4 \mathbf{Y}_{d}^{\dagger} \mathbf{m}_{d}^{2} \mathbf{Y}_{d} \mathbf{Y}_{d}^{\dagger} \mathbf{Y}_{d}-4 \mathbf{Y}_{d}^{\dagger} \mathbf{Y}_{d} \mathbf{m}_{Q}^{2} \mathbf{Y}_{d}^{\dagger} \mathbf{Y}_{d}-4 \mathbf{Y}_{d}^{\dagger} \mathbf{Y}_{d} \mathbf{Y}_{d}^{\dagger} \mathbf{m}_{d}^{2} \mathbf{Y}_{d}-2 \mathbf{Y}_{d}^{\dagger} \mathbf{Y}_{d} \mathbf{Y}_{d}^{\dagger} \mathbf{Y}_{d} \mathbf{m}_{Q}^{2}
\end{aligned}
$$




$$
\begin{aligned}
& -\left[\left(\mathbf{m}_{Q}^{2}+4 m_{H_{u}}^{2}\right) \mathbf{Y}_{u}^{\dagger} \mathbf{Y}_{u}+2 \mathbf{Y}_{u}^{\dagger} \mathbf{m}_{u}^{2} \mathbf{Y}_{u}+\mathbf{Y}_{u}^{\dagger} \mathbf{Y}_{u} \mathbf{m}_{Q}^{2}\right] \operatorname{Tr}\left(3 \mathbf{Y}_{u}^{\dagger} \mathbf{Y}_{u}\right) \\
& -\left[\left(\mathbf{m}_{Q}^{2}+4 m_{H_{d}}^{2}\right) \mathbf{Y}_{d}^{\dagger} \mathbf{Y}_{d}+2 \mathbf{Y}_{d}^{\dagger} \mathbf{m}_{d}^{2} \mathbf{Y}_{d}+\mathbf{Y}_{d}^{\dagger} \mathbf{Y}_{d} \mathbf{m}_{Q}^{2}\right] \operatorname{Tr}\left(3 \mathbf{Y}_{d}^{\dagger} \mathbf{Y}_{d}+\mathbf{Y}_{e}^{\dagger} \mathbf{Y}_{e}\right) \\
& -6 \mathbf{Y}_{u}^{\dagger} \mathbf{Y}_{u} \operatorname{Tr}\left(\mathbf{m}_{Q}^{2} \mathbf{Y}_{u}^{\dagger} \mathbf{Y}_{u}+\mathbf{Y}_{u}^{\dagger} \mathbf{m}_{u}^{2} \mathbf{Y}_{u}\right) \\
& -\mathbf{Y}_{d}^{\dagger} \mathbf{Y}_{d} \operatorname{Tr}\left(6 \mathbf{m}_{Q}^{2} \mathbf{Y}_{d}^{\dagger} \mathbf{Y}_{d}+6 \mathbf{Y}_{d}^{\dagger} \mathbf{m}_{d}^{2} \mathbf{Y}_{d}+2 \mathbf{m}_{L}^{2} \mathbf{Y}_{e}^{\dagger} \mathbf{Y}_{e}+2 \mathbf{Y}_{e}^{\dagger} \mathbf{m}_{e}^{2} \mathbf{Y}_{e}\right) \\
& -4\left\{\mathbf{Y}_{u}^{\dagger} \mathbf{Y}_{u} \mathbf{h}_{u}^{\dagger} \mathbf{h}_{u}+\mathbf{h}_{u}^{\dagger} \mathbf{h}_{u} \mathbf{Y}_{u}^{\dagger} \mathbf{Y}_{u}+\mathbf{Y}_{u}^{\dagger} \mathbf{h}_{u} \mathbf{h}_{u}^{\dagger} \mathbf{Y}_{u}+\mathbf{h}_{u}^{\dagger} \mathbf{Y}_{u} \mathbf{Y}_{u}^{\dagger} \mathbf{h}_{u}\right\} \\
& -4\left\{\mathbf{Y}_{d}^{\dagger} \mathbf{Y}_{d} \mathbf{h}_{d}^{\dagger} \mathbf{h}_{d}+\mathbf{h}_{d}^{\dagger} \mathbf{h}_{d} \mathbf{Y}_{d}^{\dagger} \mathbf{Y}_{d}+\mathbf{Y}_{d}^{\dagger} \mathbf{h}_{d} \mathbf{h}_{d}^{\dagger} \mathbf{Y}_{d}+\mathbf{h}_{d}^{\dagger} \mathbf{Y}_{d} \mathbf{Y}_{d}^{\dagger} \mathbf{h}_{d}\right\} \\
& -\mathbf{h}_{u}^{\dagger} \mathbf{h}_{u} \operatorname{Tr}\left[6 \mathbf{Y}_{u}^{\dagger} \mathbf{Y}_{u}\right]-\mathbf{Y}_{u}^{\dagger} \mathbf{Y}_{u} \operatorname{Tr}\left[6 \mathbf{h}_{u}^{\dagger} \mathbf{h}_{u}\right]-\mathbf{h}_{u}^{\dagger} \mathbf{Y}_{u} \operatorname{Tr}\left[6 \mathbf{Y}_{u}^{\dagger} \mathbf{h}_{u}\right]-\mathbf{Y}_{u}^{\dagger} \mathbf{h}_{u} \operatorname{Tr}\left[6 \mathbf{h}_{u}^{\dagger} \mathbf{Y}_{u}\right] \\
& -\mathbf{h}_{d}^{\dagger} \mathbf{h}_{d} \operatorname{Tr}\left[6 \mathbf{Y}_{d}^{\dagger} \mathbf{Y}_{d}+2 \mathbf{Y}_{e}^{\dagger} \mathbf{Y}_{e}\right]-\mathbf{Y}_{d}^{\dagger} \mathbf{Y}_{d} \operatorname{Tr}\left[6 \mathbf{h}_{d}^{\dagger} \mathbf{h}_{d}+2 \mathbf{h}_{e}^{\dagger} \mathbf{h}_{e}\right] \\
& -\mathbf{h}_{d}^{\dagger} \mathbf{Y}_{d} \operatorname{Tr}\left[6 \mathbf{Y}_{d}^{\dagger} \mathbf{h}_{d}+2 \mathbf{Y}_{e}^{\dagger} \mathbf{h}_{e}\right]-\mathbf{Y}_{d}^{\dagger} \mathbf{h}_{d} \operatorname{Tr}\left[6 \mathbf{h}_{d}^{\dagger} \mathbf{Y}_{d}+2 \mathbf{h}_{e}^{\dagger} \mathbf{Y}_{e}\right] \\
& +\frac{2}{5} g_{1}^{2}\left\{\left(2 \mathbf{m}_{Q}^{2}+4 m_{H_{u}}^{2}\right) \mathbf{Y}_{u}^{\dagger} \mathbf{Y}_{u}+4 \mathbf{Y}_{u}^{\dagger} \mathbf{m}_{u}^{2} \mathbf{Y}_{u}+2 \mathbf{Y}_{u}^{\dagger} \mathbf{Y}_{u} \mathbf{m}_{Q}^{2}+4 \mathbf{h}_{u}^{\dagger} \mathbf{h}_{u}-4 M_{1} \mathbf{h}_{u}^{\dagger} \mathbf{Y}_{u}\right. \\
& -4 M_{1}^{*} \mathbf{Y}_{u}^{\dagger} \mathbf{h}_{u}+8\left|M_{1}\right|^{2} \mathbf{Y}_{u}^{\dagger} \mathbf{Y}_{u}+\left(\mathbf{m}_{Q}^{2}+2 m_{H_{d}}^{2}\right) \mathbf{Y}_{d}^{\dagger} \mathbf{Y}_{d}+2 \mathbf{Y}_{d}^{\dagger} \mathbf{m}_{d}^{2} \mathbf{Y}_{d} \\
& \left.+\mathbf{Y}_{d}^{\dagger} \mathbf{Y}_{d} \mathbf{m}_{Q}^{2}+2 \mathbf{h}_{d}^{\dagger} \mathbf{h}_{d}-2 M_{1} \mathbf{h}_{d}^{\dagger} \mathbf{Y}_{d}-2 M_{1}^{*} \mathbf{Y}_{d}^{\dagger} \mathbf{h}_{d}+4\left|M_{1}\right|^{2} \mathbf{Y}_{d}^{\dagger} \mathbf{Y}_{d}\right\} \\
& +\frac{2}{5} g_{1}^{2} \mathcal{S}^{\prime}-\frac{128}{3} g_{3}^{4}\left|M_{3}\right|^{2}+32 g_{3}^{2} g_{2}^{2}\left(\left|M_{3}\right|^{2}+\left|M_{2}\right|^{2}+\operatorname{Re}\left[M_{2} M_{3}^{*}\right]\right) \\
& +\frac{32}{45} g_{3}^{2} g_{1}^{2}\left(\left|M_{3}\right|^{2}+\left|M_{1}\right|^{2}+\operatorname{Re}\left[M_{1} M_{3}^{*}\right]\right)+33 g_{2}^{4}\left|M_{2}\right|^{2} \\
& +\frac{2}{5} g_{2}^{2} g_{1}^{2}\left(\left|M_{2}\right|^{2}+\left|M_{1}\right|^{2}+\operatorname{Re}\left[M_{1} M_{2}^{*}\right]\right)+\frac{199}{75} g_{1}^{4}\left|M_{1}\right|^{2} \\
& +\frac{16}{3} g_{3}^{2} \sigma_{3}+3 g_{2}^{2} \sigma_{2}+\frac{1}{15} g_{1}^{2} \sigma_{1}
\end{aligned}
$$

$$
\begin{aligned}
\beta_{\mathbf{m}_{L}^{2}}^{(1)}= & \left(\mathbf{m}_{L}^{2}+2 m_{H_{d}}^{2}\right) \mathbf{Y}_{e}^{\dagger} \mathbf{Y}_{e}+2 \mathbf{Y}_{e}^{\dagger} \mathbf{m}_{e}^{2} \mathbf{Y}_{e}+\mathbf{Y}_{e}^{\dagger} \mathbf{Y}_{e} \mathbf{m}_{L}^{2}+2 \mathbf{h}_{e}^{\dagger} \mathbf{h}_{e} \\
& -6 g_{2}^{2}\left|M_{2}\right|^{2}-\frac{6}{5} g_{1}^{2}\left|M_{1}\right|^{2}-\frac{3}{5} g_{1}^{2} \mathcal{S}
\end{aligned}
$$

$$
\begin{aligned}
\beta_{\mathbf{m}_{L}^{2}}^{(2)}= & -\left(2 \mathbf{m}_{L}^{2}+8 m_{H_{d}}^{2}\right) \mathbf{Y}_{e}^{\dagger} \mathbf{Y}_{e} \mathbf{Y}_{e}^{\dagger} \mathbf{Y}_{e}-4 \mathbf{Y}_{e}^{\dagger} \mathbf{m}_{e}^{2} \mathbf{Y}_{e} \mathbf{Y}_{e}^{\dagger} \mathbf{Y}_{e}-4 \mathbf{Y}_{e}^{\dagger} \mathbf{Y}_{e} \mathbf{m}_{L}^{2} \mathbf{Y}_{e}^{\dagger} \mathbf{Y}_{e} \\
& -4 \mathbf{Y}_{e}^{\dagger} \mathbf{Y}_{e} \mathbf{Y}_{e}^{\dagger} \mathbf{m}_{e}^{2} \mathbf{Y}_{e}-2 \mathbf{Y}_{e}^{\dagger} \mathbf{Y}_{e} \mathbf{Y}_{e}^{\dagger} \mathbf{Y}_{e} \mathbf{m}_{L}^{2} \\
& -\left[\left(\mathbf{m}_{L}^{2}+4 m_{H_{d}}^{2}\right) \mathbf{Y}_{e}^{\dagger} \mathbf{Y}_{e}+2 \mathbf{Y}_{e}^{\dagger} \mathbf{m}_{e}^{2} \mathbf{Y}_{e}+\mathbf{Y}_{e}^{\dagger} \mathbf{Y}_{e} \mathbf{m}_{L}^{2}\right] \operatorname{Tr}\left(3 \mathbf{Y}_{d}^{\dagger} \mathbf{Y}_{d}+\mathbf{Y}_{e}^{\dagger} \mathbf{Y}_{e}\right) \\
& -\mathbf{Y}_{e}^{\dagger} \mathbf{Y}_{e} \operatorname{Tr}\left[6 \mathbf{m}_{Q}^{2} \mathbf{Y}_{d}^{\dagger} \mathbf{Y}_{d}+6 \mathbf{Y}_{d}^{\dagger} \mathbf{m}_{d}^{2} \mathbf{Y}_{d}+2 \mathbf{m}_{L}^{2} \mathbf{Y}_{e}^{\dagger} \mathbf{Y}_{e}+2 \mathbf{Y}_{e}^{\dagger} \mathbf{m}_{e}^{2} \mathbf{Y}_{e}\right]
\end{aligned}
$$




$$
\begin{aligned}
& -4\left\{\mathbf{Y}_{e}^{\dagger} \mathbf{Y}_{e} \mathbf{h}_{e}^{\dagger} \mathbf{h}_{e}+\mathbf{h}_{e}^{\dagger} \mathbf{h}_{e} \mathbf{Y}_{e}^{\dagger} \mathbf{Y}_{e}+\mathbf{Y}_{e}^{\dagger} \mathbf{h}_{e} \mathbf{h}_{e}^{\dagger} \mathbf{Y}_{e}+\mathbf{h}_{e}^{\dagger} \mathbf{Y}_{e} \mathbf{Y}_{e}^{\dagger} \mathbf{h}_{e}\right\} \\
& -\mathbf{h}_{e}^{\dagger} \mathbf{h}_{e} \operatorname{Tr}\left[6 \mathbf{Y}_{d}^{\dagger} \mathbf{Y}_{d}+2 \mathbf{Y}_{e}^{\dagger} \mathbf{Y}_{e}\right]-\mathbf{Y}_{e}^{\dagger} \mathbf{Y}_{e} \operatorname{Tr}\left[6 \mathbf{h}_{d}^{\dagger} \mathbf{h}_{d}+2 \mathbf{h}_{e}^{\dagger} \mathbf{h}_{e}\right] \\
& -\mathbf{h}_{e}^{\dagger} \mathbf{Y}_{e} \operatorname{Tr}\left[6 \mathbf{Y}_{d}^{\dagger} \mathbf{h}_{d}+2 \mathbf{Y}_{e}^{\dagger} \mathbf{h}_{e}\right]-\mathbf{Y}_{e}^{\dagger} \mathbf{h}_{e} \operatorname{Tr}\left[6 \mathbf{h}_{d}^{\dagger} \mathbf{Y}_{d}+2 \mathbf{h}_{e}^{\dagger} \mathbf{Y}_{e}\right] \\
& +\frac{6}{5} g_{1}^{2}\left\{\left(\mathbf{m}_{L}^{2}+2 m_{H_{d}}^{2}\right) \mathbf{Y}_{e}^{\dagger} \mathbf{Y}_{e}+2 \mathbf{Y}_{e}^{\dagger} \mathbf{m}_{e}^{2} \mathbf{Y}_{e}+\mathbf{Y}_{e}^{\dagger} \mathbf{Y}_{e} \mathbf{m}_{L}^{2}+2 \mathbf{h}_{e}^{\dagger} \mathbf{h}_{e}\right. \\
& \left.\quad-2 M_{1} \mathbf{h}_{e}^{\dagger} \mathbf{Y}_{e}-2 M_{1}^{*} \mathbf{Y}_{e}^{\dagger} \mathbf{h}_{e}+4\left|M_{1}\right|^{2} \mathbf{Y}_{e}^{\dagger} \mathbf{Y}_{e}\right\}-\frac{6}{5} g_{1}^{2} \mathcal{S}^{\prime} \\
& +33 g_{2}^{4}\left|M_{2}\right|^{2}+\frac{18}{5} g_{2}^{2} g_{1}^{2}\left(\left|M_{2}\right|^{2}+\left|M_{1}\right|^{2}+\operatorname{Re}\left[M_{1} M_{2}^{*}\right]\right)+\frac{621}{25} g_{1}^{4}\left|M_{1}\right|^{2} \\
& +3 g_{2}^{2} \sigma_{2}+\frac{3}{5} g_{1}^{2} \sigma_{1}
\end{aligned}
$$

$$
\begin{aligned}
\beta_{\mathbf{m}_{u}^{2}}^{(1)}= & \left(2 \mathbf{m}_{u}^{2}+4 m_{H_{u}}^{2}\right) \mathbf{Y}_{u} \mathbf{Y}_{u}^{\dagger}+4 \mathbf{Y}_{u} \mathbf{m}_{Q}^{2} \mathbf{Y}_{u}^{\dagger}+2 \mathbf{Y}_{u} \mathbf{Y}_{u}^{\dagger} \mathbf{m}_{u}^{2}+4 \mathbf{h}_{u} \mathbf{h}_{u}^{\dagger} \\
& -\frac{32}{3} g_{3}^{2}\left|M_{3}\right|^{2}-\frac{32}{15} g_{1}^{2}\left|M_{1}\right|^{2}-\frac{4}{5} g_{1}^{2} \mathcal{S}
\end{aligned}
$$

$$
\begin{aligned}
\beta_{\mathbf{m}_{u}^{2}}^{(2)}= & -\left(2 \mathbf{m}_{u}^{2}+8 m_{H_{u}}^{2}\right) \mathbf{Y}_{u} \mathbf{Y}_{u}^{\dagger} \mathbf{Y}_{u} \mathbf{Y}_{u}^{\dagger}-4 \mathbf{Y}_{u} \mathbf{m}_{Q}^{2} \mathbf{Y}_{u}^{\dagger} \mathbf{Y}_{u} \mathbf{Y}_{u}^{\dagger}-4 \mathbf{Y}_{u} \mathbf{Y}_{u}^{\dagger} \mathbf{m}_{u}^{2} \mathbf{Y}_{u} \mathbf{Y}_{u}^{\dagger} \\
& -4 \mathbf{Y}_{u} \mathbf{Y}_{u}^{\dagger} \mathbf{Y}_{u} \mathbf{m}_{Q}^{2} \mathbf{Y}_{u}^{\dagger}-2 \mathbf{Y}_{u} \mathbf{Y}_{u}^{\dagger} \mathbf{Y}_{u} \mathbf{Y}_{u}^{\dagger} \mathbf{m}_{u}^{2}-\left(2 \mathbf{m}_{u}^{2}+4 m_{H_{u}}^{2}+4 m_{H_{d}}^{2}\right) \mathbf{Y}_{u} \mathbf{Y}_{d}^{\dagger} \mathbf{Y}_{d} \mathbf{Y}_{u}^{\dagger} \\
& -4 \mathbf{Y}_{u} \mathbf{m}_{Q}^{2} \mathbf{Y}_{d}^{\dagger} \mathbf{Y}_{d} \mathbf{Y}_{u}^{\dagger}-4 \mathbf{Y}_{u} \mathbf{Y}_{d}^{\dagger} \mathbf{m}_{d}^{2} \mathbf{Y}_{d} \mathbf{Y}_{u}^{\dagger}-4 \mathbf{Y}_{u} \mathbf{Y}_{d}^{\dagger} \mathbf{Y}_{d} \mathbf{m}_{Q}^{2} \mathbf{Y}_{u}^{\dagger}-2 \mathbf{Y}_{u} \mathbf{Y}_{d}^{\dagger} \mathbf{Y}_{d} \mathbf{Y}_{u}^{\dagger} \mathbf{m}_{u}^{2} \\
& -\left[\left(\mathbf{m}_{u}^{2}+4 m_{H_{u}}^{2}\right) \mathbf{Y}_{u} \mathbf{Y}_{u}^{\dagger}+2 \mathbf{Y}_{u} \mathbf{m}_{Q}^{2} \mathbf{Y}_{u}^{\dagger}+\mathbf{Y}_{u} \mathbf{Y}_{u}^{\dagger} \mathbf{m}_{u}^{2}\right] \operatorname{Tr}\left[6 \mathbf{Y}_{u}^{\dagger} \mathbf{Y}_{u}\right] \\
& -12 \mathbf{Y}_{u} \mathbf{Y}_{u}^{\dagger} \operatorname{Tr}\left[\mathbf{m}_{Q}^{2} \mathbf{Y}_{u}^{\dagger} \mathbf{Y}_{u}+\mathbf{Y}_{u}^{\dagger} \mathbf{m}_{u}^{2} \mathbf{Y}_{u}\right] \\
& -4\left\{\mathbf{h}_{u} \mathbf{h}_{u}^{\dagger} \mathbf{Y}_{u} \mathbf{Y}_{u}^{\dagger}+\mathbf{Y}_{u} \mathbf{Y}_{u}^{\dagger} \mathbf{h}_{u} \mathbf{h}_{u}^{\dagger}+\mathbf{h}_{u} \mathbf{Y}_{u}^{\dagger} \mathbf{Y}_{u} \mathbf{h}_{u}^{\dagger}+\mathbf{Y}_{u} \mathbf{h}_{u}^{\dagger} \mathbf{h}_{u} \mathbf{Y}_{u}^{\dagger}\right\} \\
& -4\left\{\mathbf{h}_{u} \mathbf{h}_{d}^{\dagger} \mathbf{Y}_{d} \mathbf{Y}_{u}^{\dagger}+\mathbf{Y}_{u} \mathbf{Y}_{d}^{\dagger} \mathbf{h}_{d} \mathbf{h}_{u}^{\dagger}+\mathbf{h}_{u} \mathbf{Y}_{d}^{\dagger} \mathbf{Y}_{d} \mathbf{h}_{u}^{\dagger}+\mathbf{Y}_{u} \mathbf{h}_{d}^{\dagger} \mathbf{h}_{d} \mathbf{Y}_{u}^{\dagger}\right\} \\
& -12\left\{\mathbf{h}_{u} \mathbf{h}_{u}^{\dagger} \operatorname{Tr}\left[\mathbf{Y}_{u}^{\dagger} \mathbf{Y}_{u}\right]+\mathbf{Y}_{u} \mathbf{Y}_{u}^{\dagger} \operatorname{Tr}\left[\mathbf{h}_{u}^{\dagger} \mathbf{h}_{u}\right]+\mathbf{h}_{u} \mathbf{Y}_{u}^{\dagger} \operatorname{Tr}\left[\mathbf{h}_{u}^{\dagger} \mathbf{Y}_{u}\right]+\mathbf{Y}_{u} \mathbf{h}_{u}^{\dagger} \operatorname{Tr}\left[\mathbf{Y}_{u}^{\dagger} \mathbf{h}_{u}\right]\right\} \\
& +\left[6 g_{2}^{2}-\frac{2}{5} g_{1}^{2}\right]\left\{\left(\mathbf{m}_{u}^{2}+2 m_{H_{u}}^{2}\right) \mathbf{Y}_{u} \mathbf{Y}_{u}^{\dagger}+2 \mathbf{Y}_{u} \mathbf{m}_{Q}^{2} \mathbf{Y}_{u}^{\dagger}+\mathbf{Y}_{u} \mathbf{Y}_{u}^{\dagger} \mathbf{m}_{u}^{2}+2 \mathbf{h}_{u} \mathbf{h}_{u}^{\dagger}\right\} \\
& +12 g_{2}^{2}\left\{2\left|M_{2}\right|^{2} \mathbf{Y}_{u} \mathbf{Y}_{u}^{\dagger}-M_{2}^{*} \mathbf{h}_{u} \mathbf{Y}_{u}^{\dagger}-M_{2} \mathbf{Y}_{u} \mathbf{h}_{u}^{\dagger}\right\} \\
& -\frac{4}{5} g_{1}^{2}\left\{2\left|M_{1}\right|^{2} \mathbf{Y}_{u} \mathbf{Y}_{u}^{\dagger}-M_{1}^{*} \mathbf{h}_{u} \mathbf{Y}_{u}^{\dagger}-M_{1} \mathbf{Y}_{u} \mathbf{h}_{u}^{\dagger}\right\}-\frac{8}{5} g_{1}^{2} \mathcal{S}^{\prime} \\
& -\frac{128}{3} g_{3}^{4}\left|M_{3}\right|^{2}+\frac{512}{45} g_{3}^{2} g_{1}^{2}\left(\left|M_{3}\right|^{2}+\left|M_{1}\right|^{2}+\operatorname{Re}\left[M_{1} M_{3}^{*}\right]\right)+\frac{3424}{75} g_{1}^{4}\left|M_{1}\right|^{2} \\
& +\frac{16}{3} g_{3}^{2} \sigma_{3}+\frac{16}{15} g_{1}^{2} \sigma_{1}
\end{aligned}
$$




$$
\begin{aligned}
\beta_{\mathbf{m}_{d}^{2}}^{(1)}= & \left(2 \mathbf{m}_{d}^{2}+4 m_{H_{d}}^{2}\right) \mathbf{Y}_{d} \mathbf{Y}_{d}^{\dagger}+4 \mathbf{Y}_{d} \mathbf{m}_{Q}^{2} \mathbf{Y}_{d}^{\dagger}+2 \mathbf{Y}_{d} \mathbf{Y}_{d}^{\dagger} \mathbf{m}_{d}^{2}+4 \mathbf{h}_{d} \mathbf{h}_{d}^{\dagger} \\
& -\frac{32}{3} g_{3}^{2}\left|M_{3}\right|^{2}-\frac{8}{15} g_{1}^{2}\left|M_{1}\right|^{2}+\frac{2}{5} g_{1}^{2} \mathcal{S}
\end{aligned}
$$

$$
\begin{aligned}
& \beta_{\mathbf{m}_{d}^{2}}^{(2)}=-\left(2 \mathbf{m}_{d}^{2}+8 m_{H_{d}}^{2}\right) \mathbf{Y}_{d} \mathbf{Y}_{d}^{\dagger} \mathbf{Y}_{d} \mathbf{Y}_{d}^{\dagger}-4 \mathbf{Y}_{d} \mathbf{m}_{Q}^{2} \mathbf{Y}_{d}^{\dagger} \mathbf{Y}_{d} \mathbf{Y}_{d}^{\dagger}-4 \mathbf{Y}_{d} \mathbf{Y}_{d}^{\dagger} \mathbf{m}_{d}^{2} \mathbf{Y}_{d} \mathbf{Y}_{d}^{\dagger} \\
& -4 \mathbf{Y}_{d} \mathbf{Y}_{d}^{\dagger} \mathbf{Y}_{d} \mathbf{m}_{Q}^{2} \mathbf{Y}_{d}^{\dagger}-2 \mathbf{Y}_{d} \mathbf{Y}_{d}^{\dagger} \mathbf{Y}_{d} \mathbf{Y}_{d}^{\dagger} \mathbf{m}_{d}^{2}-\left(2 \mathbf{m}_{d}^{2}+4 m_{H_{u}}^{2}+4 m_{H_{d}}^{2}\right) \mathbf{Y}_{d} \mathbf{Y}_{u}^{\dagger} \mathbf{Y}_{u} \mathbf{Y}_{d}^{\dagger} \\
& -4 \mathbf{Y}_{d} \mathbf{m}_{Q}^{2} \mathbf{Y}_{u}^{\dagger} \mathbf{Y}_{u} \mathbf{Y}_{d}^{\dagger}-4 \mathbf{Y}_{d} \mathbf{Y}_{u}^{\dagger} \mathbf{m}_{u}^{2} \mathbf{Y}_{u} \mathbf{Y}_{d}^{\dagger}-4 \mathbf{Y}_{d} \mathbf{Y}_{u}^{\dagger} \mathbf{Y}_{u} \mathbf{m}_{Q}^{2} \mathbf{Y}_{d}^{\dagger}-2 \mathbf{Y}_{d} \mathbf{Y}_{u}^{\dagger} \mathbf{Y}_{u} \mathbf{Y}_{d}^{\dagger} \mathbf{m}_{d}^{2} \\
& -\left[\left(\mathbf{m}_{d}^{2}+4 m_{H_{d}}^{2}\right) \mathbf{Y}_{d} \mathbf{Y}_{d}^{\dagger}+2 \mathbf{Y}_{d} \mathbf{m}_{Q}^{2} \mathbf{Y}_{d}^{\dagger}+\mathbf{Y}_{d} \mathbf{Y}_{d}^{\dagger} \mathbf{m}_{d}^{2}\right] \operatorname{Tr}\left(6 \mathbf{Y}_{d}^{\dagger} \mathbf{Y}_{d}+2 \mathbf{Y}_{e}^{\dagger} \mathbf{Y}_{e}\right) \\
& -4 \mathbf{Y}_{d} \mathbf{Y}_{d}^{\dagger} \operatorname{Tr}\left(3 \mathbf{m}_{Q}^{2} \mathbf{Y}_{d}^{\dagger} \mathbf{Y}_{d}+3 \mathbf{Y}_{d}^{\dagger} \mathbf{m}_{d}^{2} \mathbf{Y}_{d}+\mathbf{m}_{L}^{2} \mathbf{Y}_{e}^{\dagger} \mathbf{Y}_{e}+\mathbf{Y}_{e}^{\dagger} \mathbf{m}_{e}^{2} \mathbf{Y}_{e}\right) \\
& -4\left\{\mathbf{h}_{d} \mathbf{h}_{d}^{\dagger} \mathbf{Y}_{d} \mathbf{Y}_{d}^{\dagger}+\mathbf{Y}_{d} \mathbf{Y}_{d}^{\dagger} \mathbf{h}_{d} \mathbf{h}_{d}^{\dagger}+\mathbf{h}_{d} \mathbf{Y}_{d}^{\dagger} \mathbf{Y}_{d} \mathbf{h}_{d}^{\dagger}+\mathbf{Y}_{d} \mathbf{h}_{d}^{\dagger} \mathbf{h}_{d} \mathbf{Y}_{d}^{\dagger}\right\} \\
& -4\left\{\mathbf{h}_{d} \mathbf{h}_{u}^{\dagger} \mathbf{Y}_{u} \mathbf{Y}_{d}^{\dagger}+\mathbf{Y}_{d} \mathbf{Y}_{u}^{\dagger} \mathbf{h}_{u} \mathbf{h}_{d}^{\dagger}+\mathbf{h}_{d} \mathbf{Y}_{u}^{\dagger} \mathbf{Y}_{u} \mathbf{h}_{d}^{\dagger}+\mathbf{Y}_{d} \mathbf{h}_{u}^{\dagger} \mathbf{h}_{u} \mathbf{Y}_{d}^{\dagger}\right\} \\
& -4 \mathbf{h}_{d} \mathbf{h}_{d}^{\dagger} \operatorname{Tr}\left(3 \mathbf{Y}_{d}^{\dagger} \mathbf{Y}_{d}+\mathbf{Y}_{e}^{\dagger} \mathbf{Y}_{e}\right)-4 \mathbf{Y}_{d} \mathbf{Y}_{d}^{\dagger} \operatorname{Tr}\left(3 \mathbf{h}_{d}^{\dagger} \mathbf{h}_{d}+\mathbf{h}_{e}^{\dagger} \mathbf{h}_{e}\right) \\
& -4 \mathbf{h}_{d} \mathbf{Y}_{d}^{\dagger} \operatorname{Tr}\left(3 \mathbf{h}_{d}^{\dagger} \mathbf{Y}_{d}+\mathbf{h}_{e}^{\dagger} \mathbf{Y}_{e}\right)-4 \mathbf{Y}_{d} \mathbf{h}_{d}^{\dagger} \operatorname{Tr}\left(3 \mathbf{Y}_{d}^{\dagger} \mathbf{h}_{d}+\mathbf{Y}_{e}^{\dagger} \mathbf{h}_{e}\right) \\
& +\left[6 g_{2}^{2}+\frac{2}{5} g_{1}^{2}\right]\left\{\left(\mathbf{m}_{d}^{2}+2 m_{H_{d}}^{2}\right) \mathbf{Y}_{d} \mathbf{Y}_{d}^{\dagger}+2 \mathbf{Y}_{d} \mathbf{m}_{Q}^{2} \mathbf{Y}_{d}^{\dagger}+\mathbf{Y}_{d} \mathbf{Y}_{d}^{\dagger} \mathbf{m}_{d}^{2}+2 \mathbf{h}_{d} \mathbf{h}_{d}^{\dagger}\right\} \\
& +12 g_{2}^{2}\left\{2\left|M_{2}\right|^{2} \mathbf{Y}_{d} \mathbf{Y}_{d}^{\dagger}-M_{2}^{*} \mathbf{h}_{d} \mathbf{Y}_{d}^{\dagger}-M_{2} \mathbf{Y}_{d} \mathbf{h}_{d}^{\dagger}\right\} \\
& +\frac{4}{5} g_{1}^{2}\left\{2\left|M_{1}\right|^{2} \mathbf{Y}_{d} \mathbf{Y}_{d}^{\dagger}-M_{1}^{*} \mathbf{h}_{d} \mathbf{Y}_{d}^{\dagger}-M_{1} \mathbf{Y}_{d} \mathbf{h}_{d}^{\dagger}\right\}+\frac{4}{5} g_{1}^{2} \mathcal{S}^{\prime} \\
& -\frac{128}{3} g_{3}^{4}\left|M_{3}\right|^{2}+\frac{128}{45} g_{3}^{2} g_{1}^{2}\left(\left|M_{3}\right|^{2}+\left|M_{1}\right|^{2}+\operatorname{Re}\left[M_{1} M_{3}^{*}\right]\right)+\frac{808}{75} g_{1}^{4}\left|M_{1}\right|^{2} \\
& +\frac{16}{3} g_{3}^{2} \sigma_{3}+\frac{4}{15} g_{1}^{2} \sigma_{1}
\end{aligned}
$$

$$
\begin{aligned}
\beta_{\mathbf{m}_{e}^{2}}^{(1)}= & \left(2 \mathbf{m}_{e}^{2}+4 m_{H_{d}}^{2}\right) \mathbf{Y}_{e} \mathbf{Y}_{e}^{\dagger}+4 \mathbf{Y}_{e} \mathbf{m}_{L}^{2} \mathbf{Y}_{e}^{\dagger}+2 \mathbf{Y}_{e} \mathbf{Y}_{e}^{\dagger} \mathbf{m}_{e}^{2}+4 \mathbf{h}_{e} \mathbf{h}_{e}^{\dagger} \\
& -\frac{24}{5} g_{1}^{2}\left|M_{1}\right|^{2}+\frac{6}{5} g_{1}^{2} \mathcal{S} \\
\beta_{\mathbf{m}_{e}^{2}}^{(2)}= & -\left(2 \mathbf{m}_{e}^{2}+8 m_{H_{d}}^{2}\right) \mathbf{Y}_{e} \mathbf{Y}_{e}^{\dagger} \mathbf{Y}_{e} \mathbf{Y}_{e}^{\dagger}-4 \mathbf{Y}_{e} \mathbf{m}_{L}^{2} \mathbf{Y}_{e}^{\dagger} \mathbf{Y}_{e} \mathbf{Y}_{e}^{\dagger}-4 \mathbf{Y}_{e} \mathbf{Y}_{e}^{\dagger} \mathbf{m}_{e}^{2} \mathbf{Y}_{e} \mathbf{Y}_{e}^{\dagger} \\
& -4 \mathbf{Y}_{e} \mathbf{Y}_{e}^{\dagger} \mathbf{Y}_{e} \mathbf{m}_{L}^{2} \mathbf{Y}_{e}^{\dagger}-2 \mathbf{Y}_{e} \mathbf{Y}_{e}^{\dagger} \mathbf{Y}_{e} \mathbf{Y}_{e}^{\dagger} \mathbf{m}_{e}^{2}
\end{aligned}
$$




$$
\begin{aligned}
& -\left[\left(\mathbf{m}_{e}^{2}+4 m_{H_{d}}^{2}\right) \mathbf{Y}_{e} \mathbf{Y}_{e}^{\dagger}+2 \mathbf{Y}_{e} \mathbf{m}_{L}^{2} \mathbf{Y}_{e}^{\dagger}+\mathbf{Y}_{e} \mathbf{Y}_{e}^{\dagger} \mathbf{m}_{e}^{2}\right] \operatorname{Tr}\left[6 \mathbf{Y}_{d}^{\dagger} \mathbf{Y}_{d}+2 \mathbf{Y}_{e}^{\dagger} \mathbf{Y}_{e}\right] \\
& -4 \mathbf{Y}_{e} \mathbf{Y}_{e}^{\dagger} \operatorname{Tr}\left[3 \mathbf{m}_{Q}^{2} \mathbf{Y}_{d}^{\dagger} \mathbf{Y}_{d}+3 \mathbf{Y}_{d}^{\dagger} \mathbf{m}_{d}^{2} \mathbf{Y}_{d}+\mathbf{m}_{L}^{2} \mathbf{Y}_{e}^{\dagger} \mathbf{Y}_{e}+\mathbf{Y}_{e}^{\dagger} \mathbf{m}_{e}^{2} \mathbf{Y}_{e}\right] \\
& -4\left\{\mathbf{h}_{e} \mathbf{h}_{e}^{\dagger} \mathbf{Y}_{e} \mathbf{Y}_{e}^{\dagger}+\mathbf{Y}_{e} \mathbf{Y}_{e}^{\dagger} \mathbf{h}_{e} \mathbf{h}_{e}^{\dagger}+\mathbf{h}_{e} \mathbf{Y}_{e}^{\dagger} \mathbf{Y}_{e} \mathbf{h}_{e}^{\dagger}+\mathbf{Y}_{e} \mathbf{h}_{e}^{\dagger} \mathbf{h}_{e} \mathbf{Y}_{e}^{\dagger}\right\} \\
& -4 \mathbf{h}_{e} \mathbf{h}_{e}^{\dagger} \operatorname{Tr}\left[3 \mathbf{Y}_{d}^{\dagger} \mathbf{Y}_{d}+\mathbf{Y}_{e}^{\dagger} \mathbf{Y}_{e}\right]-4 \mathbf{Y}_{e} \mathbf{Y}_{e}^{\dagger} \operatorname{Tr}\left[3 \mathbf{h}_{d}^{\dagger} \mathbf{h}_{d}+\mathbf{h}_{e}^{\dagger} \mathbf{h}_{e}\right] \\
& -4 \mathbf{h}_{e} \mathbf{Y}_{e}^{\dagger} \operatorname{Tr}\left[3 \mathbf{h}_{d}^{\dagger} \mathbf{Y}_{d}+\mathbf{h}_{e}^{\dagger} \mathbf{Y}_{e}\right]-4 \mathbf{Y}_{e} \mathbf{h}_{e}^{\dagger} \operatorname{Tr}\left[3 \mathbf{Y}_{d}^{\dagger} \mathbf{h}_{d}+\mathbf{Y}_{e}^{\dagger} \mathbf{h}_{e}\right] \\
& +\left[6 g_{2}^{2}-\frac{6}{5} g_{1}^{2}\right]\left\{\left(\mathbf{m}_{e}^{2}+2 m_{H_{d}}^{2}\right) \mathbf{Y}_{e} \mathbf{Y}_{e}^{\dagger}+2 \mathbf{Y}_{e} \mathbf{m}_{L}^{2} \mathbf{Y}_{e}^{\dagger}+\mathbf{Y}_{e} \mathbf{Y}_{e}^{\dagger} \mathbf{m}_{e}^{2}+2 \mathbf{h}_{e} \mathbf{h}_{e}^{\dagger}\right\} \\
& +12 g_{2}^{2}\left\{2\left|M_{2}\right|^{2} \mathbf{Y}_{e} \mathbf{Y}_{e}^{\dagger}-M_{2}^{*} \mathbf{h}_{e} \mathbf{Y}_{e}^{\dagger}-M_{2} \mathbf{Y}_{e} \mathbf{h}_{e}^{\dagger}\right\} \\
& -\frac{12}{5} g_{1}^{2}\left\{2\left|M_{1}\right|^{2} \mathbf{Y}_{e} \mathbf{Y}_{e}^{\dagger}-M_{1}^{*} \mathbf{h}_{e} \mathbf{Y}_{e}^{\dagger}-M_{1} \mathbf{Y}_{e} \mathbf{h}_{e}^{\dagger}\right\} \\
& +\frac{12}{5} g_{1}^{2} \mathcal{S}^{\prime}+\frac{2808}{25} g_{1}^{4}\left|M_{1}\right|^{2}+\frac{12}{5} g_{1}^{2} \sigma_{1} .
\end{aligned}
$$

\section{Conclusion}

In this paper, we have presented the two-loop renormalization group equations for all couplings in a general softly-broken supersymmetric model, and in particular for the MSSM. If the sparticles predicted by the MSSM are found and their spectrum is determined with some accuracy (for example at an $e^{+} e^{-}$collider[33]), these results may be useful in discriminating between various candidate organizing principles for the soft supersymmetrybreaking terms at some very high input scale. One can run the parameters down to low energies to predict the masses of the sparticle spectrum and other features of low energy phenomenology in terms of what may turn out to be only a few input parameters. The masses of the sparticles depend primarily on just those soft supersymmetry-breaking couplings whose $\beta$-functions have been given to two loops here. We find that the twoloop $\beta$-functions generally make a difference of several percent (compared to the one-loop predictions) for the sparticle masses, although it is quite difficult to make meaningful estimates of the size of the two-loop corrections without committing to a specific model. At the same level of accuracy, one must also be careful to take into account threshold effects as well as the distinction between running masses and pole masses. In extensions of the MSSM which have a large non-minimal particle content above the electroweak scale, 
the two-loop corrections and threshold effects are potentially much larger.

Note added. Since the original preprint version of this paper appeared, the $\beta$ functions of Sec. II have been calculated by Yamada [34] using superfield techniques, and by Jack and Jones [35] working directly in DRED. Our results agreed with theirs except for discrepancies in the two-loop $\beta$ function (2.20) for scalar masses of the $\left(m^{2}\right)_{i}^{j}$ type, namely the coefficient of the last term proportional to $\delta_{i}^{j} g^{4} C(i)\left(\operatorname{Tr}\left[S(r) m^{2}\right]-C(G) M M^{\dagger}\right)$ and a possible dependence on the unphysical mass of the $\epsilon$ scalar. As emphasized in [35], one should properly allow the $\epsilon$ scalars to have masses and mass counterterms in the component field approach to DRED, and the result given there is correct. However, one can treat the subtractions in $\overline{\mathrm{DR}}$ in such a way that the two-loop $\beta$ functions do not depend on the unphysical $\epsilon$ scalar mass, and so that the expressions relating the pole masses to the running masses of the scalars also do not depend on the $\epsilon$ scalar masses. (This is related to the prescription used in [35] by a simple coupling constant redefinition for the scalar masses, of the type mentioned there.) The corrected results we have given here correspond to this prescription, and we are now in agreement with the authors of [34] and [35], to whom we are grateful for consultations. The subtleties involved will be reported on elsewhere. The results in the special case of the MSSM have also been corrected accordingly.

\section{Acknowledgments}

S.P.M. is indebted to Diego Castaño for many helpful discussions. M.T.V. would like to thank the theory group at the University of Southampton for their hospitality while part of this work was carried out. We are both grateful to Ian Jack, Tim Jones and Youichi Yamada for their help in correcting Eq. (2.20), and we are further indebted to Jack and Jones for pointing out the correct ordering of matrices in some of the terms in the $\beta$ functions of Sec. IV. This work was supported in part by the National Science Foundation grants PHY-90-01439 and PHY-93-06906 and U. S. Department of Energy grant DE-FG02-85ER40233. 


\section{References}

1. For reviews, see H. P. Nilles, Phys. Rep. 110, 1, (1984) or H. E. Haber and G. L. Kane, Phys. Rep. 117, 75, (1985).

2. L. Girardello and M. T. Grisaru, Nucl. Phys. B194, 65, (1982).

3. P. Langacker, in Proceedings of the PASCOS90 Symposium, Eds. P. Nath and S. Reucroft, (World Scientific, Singapore 1990) J. Ellis, S. Kelley, and D. Nanopoulos, Phys. Lett. 260B, 131, (1991); U. Amaldi, W. de Boer, and H. Furstenau, Phys. Lett. 260B, 447, (1991); P. Langacker and M. Luo, Phys. Rev. D44, 817, (1991).

4. A. Chamseddine, R. Arnowitt and P. Nath, Phys. Rev. Lett. 49, 970, (1982); H. P. Nilles, Phys. Lett. 115B, 193, (1982); L. E. Ibáñez, Phys. Lett. 118B, 73, (1982); R. Barbieri, S. Ferrara, and C. Savoy, Phys. Lett. 119B, 343, (1982); L. Hall, J. Lykken and S. Weinberg, Phys. Rev. D27, 2359, (1983); P. Nath, R. Arnowitt and A. H. Chamseddine, Nucl. Phys. B227, 121, (1983).

5. A. Lahanas and D. Nanopoulos, Phys. Rep. 145, 1, (1987).

6. G. G. Ross and R. G. Roberts, Nucl. Phys. B377, 571, (1992).

7. L. E. Ibáñez and G. G. Ross, "Electroweak Breaking in Supersymmetric Models", CERN-TH.6412/92, in Perspectives in Higgs Physics, edited by G. Kane (World Scientific, Singapore, 1993).

8. R. Arnowitt and P. Nath, SSCL-Preprint-229 (1993); Phys. Rev. Lett. 69, 725, (1992); Phys. Rev. D46, 3981, (1992); P. Nath and R. Arnowitt, Phys. Lett. B289, 368, (1992); ibid, B287 (1992) 89; Phys. Rev. Lett. 70, 3696, (1993).

9. S. Kelley, J. Lopez, D. Nanopoulos, H. Pois, and K. Yuan, Phys. Lett. B273, 423, (1991); Nucl. Phys. B398, 3, (1993); J. Lopez, D. Nanopoulos, and A. Zichichi, Phys. 
Lett. , B291, 255, (1992); J. Lopez, D. Nanopoulos, and H.Pois, Phys. Rev. D47, 2468, (1993).

10. M. Drees and M. Nojiri, Phys. Rev. D47, 376, (1993).

11. T. Elliott, S. F. King, and P. L. White, Phys. Lett. B305, 71, (1993); Phys. Lett. B314, 56, (1993); "Radiative Corrections to Higgs Boson Masses in the Next to Minimal Supersymmetric Standard Model", SHEP-92/93-21, (hep-ph 9308309).

12. R. G. Roberts and L. Roszkowski, Phys. Lett. B309, 329, (1993).

13. S. P. Martin and P. Ramond, Phys. Rev. D48, 5365, (1993).

14. P. Ramond, "Renormalization Group Study of the Minimal Supersymmetric Standard Model: No Scale Models", invited talk at workshop "Recent Advances in the Superworld", Houston TX, April 1993; D. J. Castaño, E. J. Piard, and P. Ramond, "Renormalization group study of the standard model and its extensions: II. The minimal supersymmetric standard model." University of Florida preprint UFIFTHEP-93-18, (hep-ph 9308335), August 1993.

15. W. de Boer, R. Ehret, and D. I. Kazakov, "Constraints on SUSY Masses in Supersymmetric Grand Unified Theories", IEKP-KA-93-13, (hep-ph 9308238), August 1993.

16. V. Barger, M. S. Berger, and P. Ohmann, Phys. Rev. D 47, 1093, (1993); "The Supersymmetric Particle Spectrum", Wisconsin preprint MAD-PH-801, (hep-ph 9311269), November 1993.

17. G. L. Kane, C. Kolda, L. Roszkowski, and J. D. Wells, "Study of Constrained Minimal Supersymmetry", Michigan preprint UM-TH-93-24.

18. D. R. T. Jones, Nucl. Phys. B87, 127, (1975); D. R. T. Jones and L. Mezincescu, 
Phys. Lett. 136B, 242, (1984).

19. P. West, Phys. Lett. 137B, 371, (1984); D. R. T. Jones and L. Mezincescu, Phys. Lett. 138B, 293, (1984).

20. S. P. Martin and M. T. Vaughn, Phys. Lett. 318B, 331, (1993).

21. Y. Yamada, Phys. Rev. Lett. 72, 25, (1994).

22. M. E. Machacek and M. T. Vaughn, Nucl. Phys. B222, 83, (1983).

23. M. E. Machacek and M. T. Vaughn, Nucl. Phys. B236, 221, (1984).

24. M. E. Machacek and M. T. Vaughn, Nucl. Phys. B249, 70, (1984).

25. G. 't Hooft and M. Veltman, Nucl. Phys. B44, 189, (1972).

26. W. Siegel, Phys. Lett. 84B, 193, (1979); D. M. Capper, D. R. T. Jones and P. van Nieuwenhuizen, Nucl. Phys. B167, 479, (1980).

27. W. A. Bardeen, A. J. Buras, D. W. Duke and T. W. Muta, Phys. Rev. D18, 3998, (1978).

28. I. Jack, D. R. T. Jones and K. L. Roberts, Z. Phys. C62, 161, (1994); "Equivalence of Dimensional Reduction and Dimensional Regularisation", Liverpool preprint LTH3291994.

29. I. Jack, Phys. Lett. 147B, 405, (1984) and I. Jack and H. Osborn, Nucl. Phys. B249, $472,(1985)$.

30. J. Wess and B. Zumino, Phys. Lett. 49B, 52, (1974); J. Iliopoulos and B. Zumino, Nucl. Phys. B76, 310, (1974); S. Ferrara, J. Iliopoulos and B. Zumino, Nucl. Phys. B77, 413, (1974); B. Zumino, Nucl. Phys. B89, 535, (1975); S. Ferrara and 
O. Piguet, Nucl. Phys. B93, 261, (1975); M. Grisaru, W. Siegel, and M. Rocek, Nucl. Phys. B159, 429, (1979).

31. P. S. Howe, K. S. Stelle, and P. West, Phys. Lett. 124B, 55, (1983).

32. A. Parkes and P. West, Phys. Lett. 127B, 353, (1983); J.-M. Frère, L. Mezincescu, and Y.-P. Yao, Phys. Rev. D29, 1196, (1984); Phys. Rev. D30, 2238, (1984).

33. See for instance, "JLC-I", KEK report 92-16, the JLC group, December 1992, and references contained therein.

34. Y. Yamada, "Two-loop renormalization group equations for soft SUSY breaking scalar interactions: supergraph method", KEK-TH-383, KEK Preprint 93-182, UT665, January 1994.

35. I. Jack and D. R. T. Jones, "Soft supersymmetry breaking and finiteness" Liverpool preprint LTH-334, May 1994. 